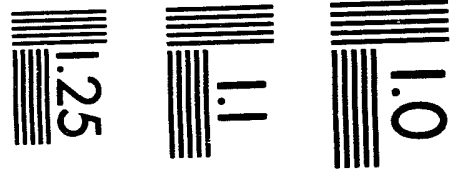

$$
\begin{aligned}
& \text { 吾至 } \\
& \text { IIII) }
\end{aligned}
$$

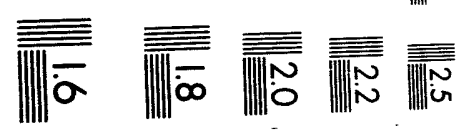



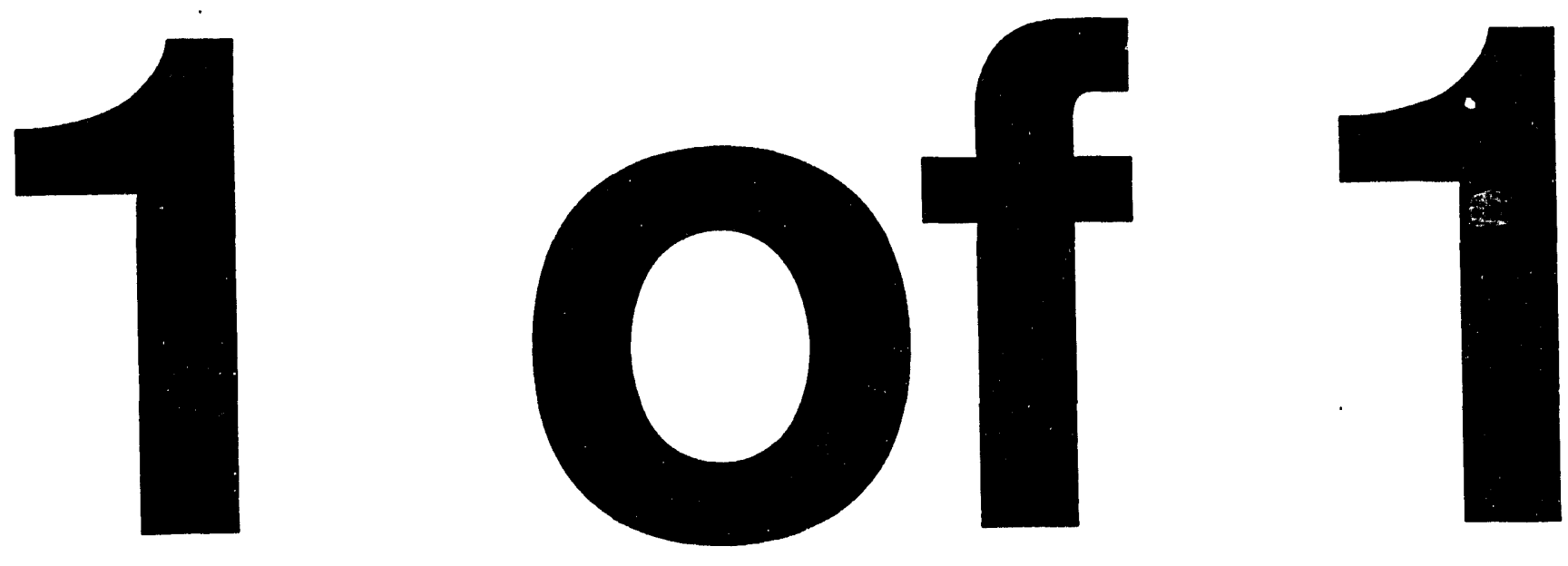


\section{Conf-9309321--1}

LA-UR- 93- 4344

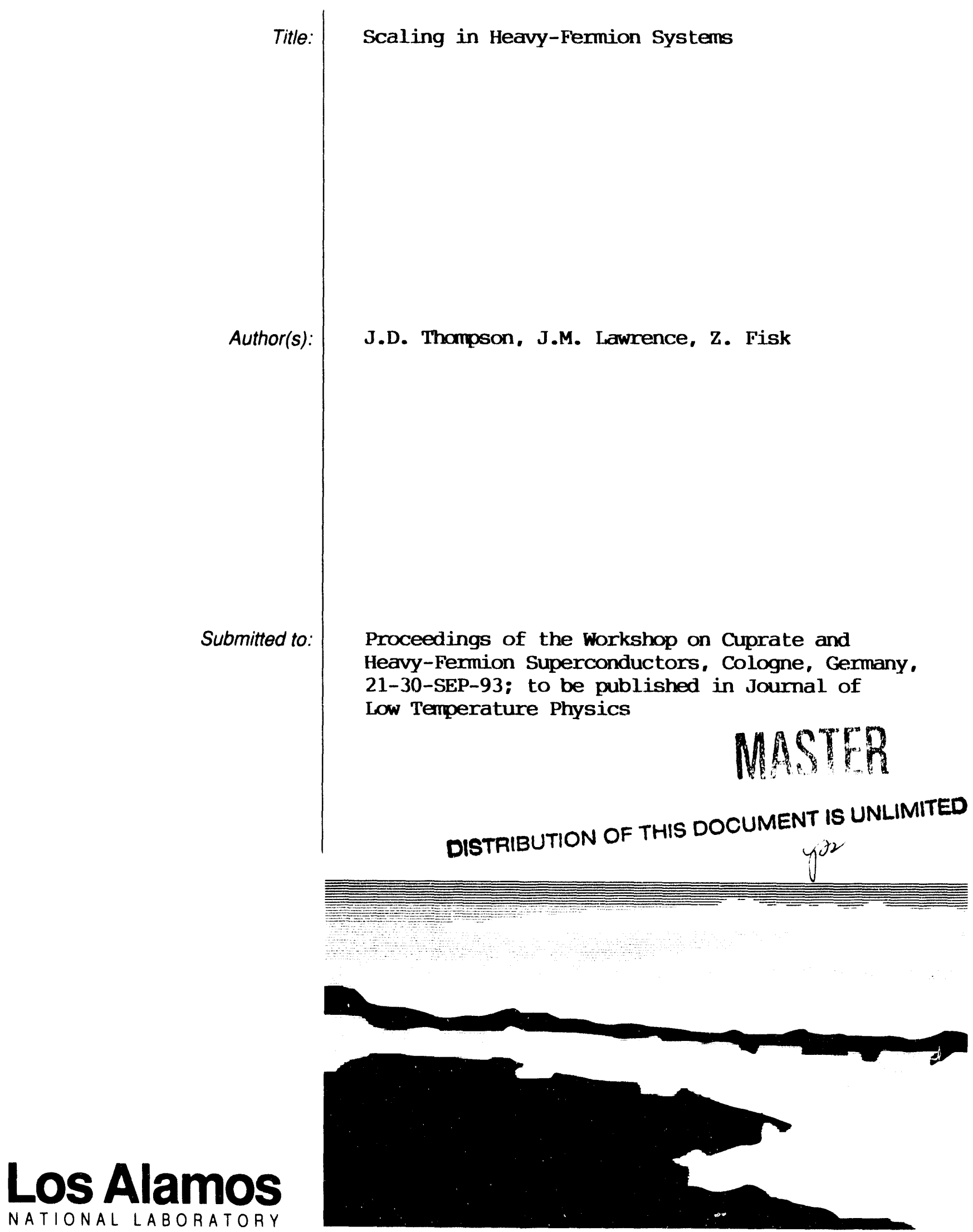

Los Alamos National Laboratory, an affirmative action/equal opportunity emplóyer, is operated by the University of California for the U.S. Department of Energy under contract W-7405-ENG-36. By acceptance of this article, the publisher recognizes that the U.S. Government retains a nonexclusive, royalty-free license to publish or reproduce the published form of this contribution, or to allow others to do so, for U.S. Government purposes. The Los Alamos National Laboratory requests that the publisher identify this article as work performed under the auspices of the U.S. Department of Energy 


\title{
Scaling in Heavy-Fermion Systems
}

\author{
J.D. Thompson, J.M. Lawrence* and Z. Fisk \\ Los Alamos National Laboratory, Los Alamos, NM 87545 \\ *Department of Physics, University of California, Irvine, CA 92717
}

\begin{abstract}
Ambient pressure characteristics of heavy-fermion compounds, such as the ratio of the low temperature magnetic susceptibility to the electronic specific heat coefficient $\gamma$ and a simple relationship between the $\mathrm{T}^{2}$-coefficient of resistivity and $\gamma^{2}$, suggest that a single-energy scale $\mathrm{T}_{0}$ dictates the physics of these materials. Such is the case for Kondo-impurity systems to which heavy-fermions are related. We consider the consequences of assuming that the electronic free energy is given by a universal function of $T / T_{0}(V)$ where $V$ is the molar volume. We show that volume-dependent magnetic susceptibility, specific heat and electrical resistivity of some heavyfermion compounds scale as $\mathrm{T} / \mathrm{T}_{\mathrm{O}}(\mathrm{V})$, at le ast over a range in pressures and temperatures. $\mathrm{A}$ further consequence of the principle assumption is that Gruneisen parameters defined as $-\partial \ell n X / \partial \ell n V$, where $\mathrm{X}$ is some physical property, should be identical for all properties and equal to that determined from electronic contributions to the volume-thermal expansion coefficient and specific heat. In several materials, this equality holds, at least approximately. Although evidence is found for single-energy scaling in heavy-fermion materials, we cannot conclude unambiguously that the basic assumption is correct in detail.
\end{abstract}

PACS numbers: $71.27 .+\mathrm{a}, 71.28 .+\mathrm{d}, 75.20 \mathrm{Hr}, 62.50+\mathrm{p}$

\section{DISCLAIMER}

\footnotetext{
This report was prepared as an account of work sponsored by an agency of the United States Government. Neither the United States Government nor any agency thereof, nor any of their employees, makes any warranty, express or implied, or assumes any legal liability or responsibility for the accuracy, completeness, or usefulness of any information, apparatus, product, or process disclosed, or represents that its use would not infringe privately owned rights. Reference herein to any specific commercial product, process, or service by trade name, trademark, manufacturer, or otherwise does not necessarily constitute or imply its endorsement, recommendation, or favoring by the United States Government or any agency thereof. The views and opinions of authors expressed herein do not necessarily state or reflect those of the United States Government or any agency thereof.
} 


\section{INTRODUCTION}

Thermodynamic properties of a Kondo impurity are determined by a single-energy scale, the Kondo temperature $T_{K}$. This characteristic of Kondo-impurity systems follows because the electronic free energy $F_{e}$ is a universal function of the ratio $T / T_{K}$, i.e. $F_{e}=-N k B T f\left(T / T_{K}\right){ }^{1}$ If the volume dependence of $F_{e}$ comes only from the volume dependence of $T_{K}$, i.e. $F_{e}(T, V)=$ $\mathrm{Fe}\left(\mathrm{T} / \mathrm{T}_{\mathrm{K}}(\mathrm{V})\right)$, then by Maxwell's relations electronic contributions to the volume-thermal expansion coefficient $\beta_{e}$ and specific heat $C_{p}$ are simply related through $\beta_{e}=\Omega_{e} C_{p} / V B T$, where $\mathrm{V}$ is the volume, $\mathrm{BT}$ is the isothermal bulk modulus, and $\Omega_{\mathrm{e}}$ is the electronic Gruneisen parameter ${ }^{2}$ given by $\Omega_{\mathrm{e}}=-\partial \ell n T_{K} / \partial \ell n V$. Therefore, it is possible to obtain the volume dependence of $T_{K}$ through measurements of $\beta_{e}$ and $C_{p}$ at 1 bar, provided $V$ and $B T$ are known. Measurements of the pressure derivatives of the Sommerfeld coefficient $\gamma=C_{p} / T$, low temperature magnetic susceptibility $\chi$ and the electrical resistivity $\rho$ also permit determination of the Gruneisen parameter through their simple relationship to $\mathrm{T}_{\mathrm{K}}$, namely $\gamma \sim 1 / \mathrm{T}_{\mathrm{K}}, \chi \sim 1 / \mathrm{T}_{\mathrm{K}}$ and $\rho=\rho(0)\left(1-\mathrm{AT}^{2}\right)$ with $\mathrm{A} \sim$ $1 / \mathrm{TK}_{\mathrm{K}}^{2}$. That is, if we define parameter $\Omega_{\mathrm{C}} \equiv \partial \ell \mathrm{n} \gamma / \partial \ell \mathrm{nV}, \Omega_{\chi} \equiv \partial \ell \mathrm{n} \chi(0) / \partial \ell \mathrm{nV}$ and $\Omega_{\mathrm{A}} \equiv$ $1 / 2 \partial \ell \mathrm{nA} / \partial \ell \mathrm{nV}$, then $\Omega_{\mathrm{C}}=\Omega \chi=\Omega_{\mathrm{A}}=\Omega_{\mathrm{e}}$. In principle, equality of these Gruneisen parameters could be determined experimentally, but this has not been done. The reason is that in the impurity limit, i.e. parts per million impurities embedded in a non-magnetic host, the pressure dependence of the impurity contribution is dominated by that of the host material so that one has to subtract two comparable values, which can lead to substantial error. The only systematic study of Kondoimpurity Gruneisen parameters has come from pressure-dependent resistivity measurements. Schilling ${ }^{3}$ has shown for dilute Ce impurities in several different non-magnetic hosts that $\Omega_{\mathrm{A}}$ is very large, ranging from 29 to 134 , and that generally larger values of $\Omega_{A}$ correspond to smaller values of $\mathrm{TK}_{\mathrm{K}}$.

For heavy-fermion compounds, which contain a periodic array of $\sim 10^{23} \mathrm{Ce}, \mathrm{Yb}$ or $\mathrm{U}$ atoms, measurements of physical properties such as $\chi(T), \rho(T)$ and the inelastic neutron lineshape suggest that at moderately high temperatures these materials can be treated as a collection of noninteracting Kondo impurities. ${ }^{4}$ In the limit of very low temperatures, several facts point to the dominance of a single-energy scale. One is that the Wilson ratio $\mathrm{R}$, which is the normalized ratio of $\chi(0)$ to $\gamma$, is very close to unity 5 for many heavy-fermion compounds and seldom exceeds two; hence, the susceptibility and specific heat are equally enhanced by the many-body interactions responsible for the heavy-fermion groundstate. The second is that the thermal coefficient $A$ of the resistivity $\left(\rho(T)=\rho(0)+A T^{2}\right.$ for atomically ordered heavy-fermions) is proportional to $\gamma^{2}$ for a large number of heavy-fermion-systems. ${ }^{6}$ These facts can be understood by assuming that $\gamma \sim \chi(0) \sim 1 / \mathrm{T}_{\mathrm{O}}$ and $\mathrm{A} \sim 1 / \mathrm{T}_{\mathrm{O}}{ }^{2}$, i.e. a single-energy scale $\mathrm{T}_{\mathrm{O}}$ dominates at low temperature.

For non-interacting Kondo impurities, theory ${ }^{7}$ predicts that the Wilson ratio is equal to $1+\left(1 /\left(N_{f}-1\right)\right)$ where $N_{f}$ is the orbital degeneracy of the impurity; hence, $R$ varies from 2 for spin$1 / 2$ to 1 for large $N_{f}$. Because values of $R$ for the compounds are comparable to this, the interpretation is often given that the low temperature scale $\mathrm{T}_{\mathrm{O}}$ is equal to the single-ion Kondo 
temperature $T_{K}$. Of course, it is clearly understood that the two cases contain an essential difference: the resistivity for the impurity case decreases from the low ternperature value $\rho(0)$ as $\rho(T)=\rho(0)\left(1-T / T_{K}{ }^{2}\right)$ and $\rho(0)$ is the maximum allowed by the unitarity limit; whereas, for the compounds the resistivity increases with temperature as $\rho(0)+A T^{2}$, where $\rho(0)$ is a small value typical of simple metals but $A$ is larger by factors of $10^{4}$ to $10^{6}$ than found for electron-electron scattering in simple metals. ${ }^{4}$ An interpretation of this difference ${ }^{8}$ in behaviors is that Bloch states are formed at low temperatures in heavy-fermions due to site-to-site coherence in phase shifts produced by resonant scattering of conduction electrons by the heavy-fermion $\mathrm{f}$-spins. It seems likely that coherence may be mediated by Ruderman-Kittel-Kasuya-Yosida (RKKY) interactions between partially Kondo-compensated spins of the local-moment impurities. Although in particular materials either Kondo-impurity or RKKY interactions may dominate, these two interactions must be present, at a minimum, in materials containing a periodic array of Kondoions. ${ }^{9}$ For RKKY interactions sufficiently strong relative to Kondo-like interactions, the ground state of the lattice will be antiferromagnetic (or possibly ferromagnetic). At the other extreme, Kondo compensation leads to a non-magnetic groundstate. For large $\mathrm{T}_{\mathrm{K}} \gg 100 \mathrm{~K}$ this groundstate is typically mixed-valent; but for heavy-fermions with $T_{K} \ll 100 \mathrm{~K}$, antiferromagnetic correlations are present in the non-magnetic groundstate. Indeed, heavy-fermion behavior typically occurs in a regime where these two energy scales, $T_{K}$ and $T_{R K K Y}$, are comparable. Therefore, there is no a priori justification for the assumption that a single-energy scale dominates the thermodynamics of heavy-fermion compounds.

The contradiction between the belief that two energy scales should be present, and the fact that the low temperature behavior of $C_{p}, \chi$ and $\rho$ appears to be dominated by a single scale $T_{0}$, is only apparent. The general expectation ${ }^{10}$ is that there exists a coherence temperature, $T_{\text {coh }}$, which is set by an interplay between TRKKY and $\mathrm{T}_{\mathrm{K}}$, and below which the resistivity varies as $\mathrm{AT}^{2}$ and the quantities $1 / \gamma, 1 / \chi(0)$ and $1 / \sqrt{\mathrm{A}}$ are all proportional to a low temperature scale $\mathrm{T}_{0}$. Antiferromagnetic correlations, when present, also should be important only at these low temperatures. For higher temperatures, the single-ion temperature $T_{K}$ sets the scale.

The central issue is whether $T_{O}$ and $T_{K}$ are fundamentally different. If so, there should be two observable ranges of temperature, one where the electronic free energy scales as $-\mathrm{NkBTf}\left(\mathrm{T} / \mathrm{T}_{\mathrm{O}}\right)$ and one where it scales as $-\mathrm{NkB} \mathrm{Tf}\left(\mathrm{T} / \mathrm{T}_{\mathrm{K}}\right)$. A clear signature that $\mathrm{T}_{\mathrm{O}}$ and $\mathrm{T}_{\mathrm{K}}$ differ will be that the Gruneisen parameters $-\partial \ell n T_{O} / \partial \ell n V$ and $-\partial \ell n T_{K} / \partial \ell n V$ are not equal. If this occurs we will speak of two-energy scaling; but if the Gruneisen parameter has the same value at all temperatures, we will say that the system exhibits single-energy scaling. Of course, to determine $\Omega_{e}$, phonon and crystal-field contributions will have to be determined and subtracted.

Nevertheless, because heavy-fermions are a dense lattice of Kondo ions, then unlike the impurity case, the Gruneisen parameters are measurable, making possible a quantitative test of the freeenergy scaling assumption. 


\section{SCALING OF PHYSICAL PROPERTIES}

There are three ways the Gruneisen parameter can be determined: (1) From pressuredependent measurements of $\chi(0), \gamma$ or $\rho \sim \mathrm{AT}^{2}, \Omega_{\chi}, \Omega_{\mathrm{C}}$ or $\Omega_{\mathrm{A}}$ can be determined in the $\mathrm{T} \rightarrow 0$ limit. (2) After subtraction of phonons and crystal-field effects, $\Omega_{\mathrm{e}}$ can be determined as a function of temperature from the ratio $\beta_{\mathrm{e}}(\mathrm{T}) \mathrm{VB} / \mathrm{C}_{\mathrm{p}}(\mathrm{T})$. (3) In any temperature interval where $\chi$, $\rho$ or $\mathrm{C}_{\mathrm{p}}$ exhibit scaling in the sense that data obtained at different pressures collapse onto a universal curve $\Omega$ can be determined over the temperature range where the data scale. In this section we examine evidence for scaling in this latter sense, considering in turn the susceptibility, resistivity and specific heat.

Figure 1(a) shows the $4 \mathrm{f}$ contribution to the magnetic susceptibility of $\mathrm{CeSn}_{3}$ at several pressures plotted as a function of temperature. ${ }^{11}$ At 1 bar the susceptibility has a maximum at $T_{\mathrm{m}} \approx 150 \mathrm{~K}$. With increasing pressure $T_{\mathrm{m}}$ moves to higher temperatures and $\chi(T)$ decreases. As shown in Fig. 1(b), if $\chi(T)$ normalized to its maximum value $\chi_{\mathrm{m}} \equiv \chi\left(\mathrm{T}_{\mathrm{m}}\right)$ is plotted as a function of reduced temperature $T / T_{m}(P)$, the data of Fig. 1(a) collapse onto a single curve. Scaling of these data holds very well except at the lowest temperatures and highest pressure and even here deviation from a universal curve is less than $10 \%$. For non-interacting Kondo impurities a maximum in $\chi$ is expected, ${ }^{12}$ provided the ground-state degeneracy $\mathrm{N}_{\mathrm{f}}$ is greater than 2 . In the absence of crystal-field splitting, the total angular momentum of $\mathrm{Ce}^{3+}$ is $\mathrm{J}=5 / 2$, which is the case for $\mathrm{CeSn} 3$, and, therefore, $\mathrm{N}_{\mathrm{f}}=2 \mathrm{~J}+1=6$. For this degeneracy Rajan ${ }^{12}$ has shown that $\mathrm{T}_{\mathrm{m}} \approx 1 / 3 \mathrm{TK}_{\mathrm{K}}$. The susceptibility thus has the same form and exhibits the same scaling property expected of a single-ion Kondo impurity with $\mathrm{T}_{\mathrm{K}} \approx 450 \mathrm{~K}$. For the lower pressures, scaling appears to hold from $T=0$ to $T=2 / 3 T_{K}$; for the highest pressure, single-energy scaling seems to break down at approximately $0.1 \mathrm{TK}_{\mathrm{K}}$. However, exact behavior at the lowest temperatures is subject to two uncertainties: it depends on assumptions concerning subtraction of a Curie-like contribution arising from impurities and on the assumptions that the non- $4 \mathrm{f}$ contribution can be estimated from the susceptibility of $\mathrm{LaSn}_{3}$ and that it is pressure independent. ${ }^{11}$ Scaling below about $10 \mathrm{~K}$ is uncertain to the extent these assumptions are uncertain.

Comparably good scaling of pressure-dependent susceptibility data is found ${ }^{13}$ for $\mathrm{YbCuAl}$ $(\mathrm{N}=8)$ in which $\mathrm{T}_{\mathrm{m}}(\mathrm{P}=0)=27 \mathrm{~K}$, although again scaling is somewhat uncertain below $0.5 \mathrm{Tm}$. In this case, though, $\partial \mathrm{T}_{\mathrm{m}} / \partial \mathrm{P}<0$ and $\partial \chi(\mathrm{T}=0) / \partial \mathrm{P}>0$. As will be discussed later, opposite pressure dependencies of physical properties of $\mathrm{Ce}$ - and $\mathrm{Yb}$-based heavy-fermion compounds are a general feature that can be understood qualitatively from pressure-induced enhancement of $4 \mathrm{f}$-conduction electron hybridization in Ce compounds but reduced hybridization in $\mathrm{Yb}$ materials.

The heavy-fermion superconductor UPt 3 also exhibits a maximum in susceptibility near $18 \mathrm{~K}$ for a magnetic field applied in the basal plane of the hexagonal crystals, Fig. 2(a). For fields along the c-axis, $\chi(T)$ is only weakly temperature dependent. ${ }^{14}$ The origin of the basal-plane maximum most likely arises from antiferromagnetic correlations that appear to be responsible for a metamagnetic transition which occurs at $21 \mathrm{~T} .{ }^{15}$ Figure 2(a) shows that this maximum moves to 
higher temperatures and the magnitude of $\chi(T)$ decreases with increasing pressure. As with CeSn3, plotting $\chi / \chi_{\mathrm{m}}$ versus $\mathrm{T} / \mathrm{T}_{\mathrm{m}}$ scales the pressure-dependent data onto a single curve (Fig. 2(b)). In this case, no background contribution has been subtracted but presumably it is relatively small compared to the $5 f$ contribution. Scaling holds very well over a range $0.3 \mathrm{~T}_{\mathrm{m}}<\mathrm{T}<3 \mathrm{~T} \mathrm{~m}$. Because the resistivity of UPt 3 varies as $\mathrm{T}^{2}$ only below about $2 \mathrm{~K}$, it is not known whether susceptibility scaling extends into the fully coherent regime.

Like UPt3, $\mathrm{CeRu}_{2} \mathrm{Si}_{2}$ also exhibits a metamagnetic transition, only at a field, 8T, applied along the tetragonal c-axis. ${ }^{16}$ Inelastic neutron-scattering experiments ${ }^{17}$ show that this metamagnetic transition arises from field-induced collapse of intersite magnetic correlations that are incommensurate with the crystal lattice. The proximity to this transition produces a maximum in the c-axis susceptibility near $T_{m}=10 \mathrm{~K}$ that is shifted to higher temperatures and becomes less pronounced with applied pressure. ${ }^{18}$ Mignot et al. ${ }^{19}$ have found that these pressure-dependent data also scale simply onto a universal curve, as discussed $\mathrm{fc}_{\mathrm{C}} \mathrm{CeSn} 3$, with scaling observed over the range $0.2 \mathrm{~T}_{\mathrm{m}}<\mathrm{T}<4 \mathrm{~T}_{\mathrm{m}}$. It is significant that, unlike CeSn3, the groundstate of $\mathrm{CeRu}_{2} \mathrm{Si}_{2}$ is only doubly degenerate. Therefore, scaling of magnetic susceptibility exists in Ce-based heavyfermion compounds irrespective of groundstate degeneracy as well as in $\mathrm{Yb}$ - and $\mathrm{U}$-based systems. It also occurs for very different groundstates: mixed valence or heavy-fermions with strong antiferromagnetic correlations. However, for the latter case the question remains whether scaling is valid at temperatures (of order $0.1 \mathrm{~T}$ ) where the existence of a $\mathrm{T}^{2}$ variation in the resistivity indicates a fully coherent groundstate; hence, it is not established whether single-energy scaling of the susceptibility is valid for this case, as it appears to be for CeSn3.

Given the observations that the $\mathrm{T}^{2}$-coefficient of resistivity is proportioned to $\gamma^{2}$ and that the Wilson ratio is near unity for a large number of heavy-fermion compounds, we expect scaling for the low temperature resistivity of these materials. Shown in Fig. 3(a) is the temperaturedependent resistivity of $\mathrm{CeSn} 3$ at various pressures. Between approximately 20 and $50 \mathrm{~K}$ the resistivity varies as $\rho(0)+A T^{2}$. Defining $T_{O}=1 / \sqrt{A}\left(T_{O} \approx 30 K\right)$, we test for scaling by plotting $\rho$ vs. T/To in Fig. 3(b). Scaling is valid below about $50 \mathrm{~K}$, but deviations are observed at higher temperatures and also at temperatures below about $20 \mathrm{~K}$ (Fig. 4(a)). The latter can be seen (Fig. $4(\mathrm{~b})$ ) to be related to the fact that below $20 \mathrm{~K} \rho(\mathrm{T})=\rho(0)+\mathrm{bT}^{3}$. This $\mathrm{T}^{3}$ behavior is not expected for a heavy-mass Fermi Liquid and is not understood. (We have observed it at $\mathrm{P}=0$ for polycrystalline samples having a range of stoichiometries between $\mathrm{CeSn} 2.90$ and $\mathrm{CeSn} 3.05$ and have determined that the coefficient $b$ is independent of magnetic field in the range $0-8$ Tesla). There could be several reasons for deviations from scaling above $50 \mathrm{~K}$ : (1) At intermediate temperatures phonons should begin to contribute non-trivially to conduction-electron scattering. This contribution has not been subtracted from the data. (2) Non-isobaric conditions arise above $-50 \mathrm{~K}$ due to thermal expansion of the sample and of pressure-cell components. (This, of course, is also a factor for susceptibility measurements made over a wide temperature interval but does not 
appear to have a significant effect on susceptibility scaling, vis-a-vis Fig. 1(b)). (3) Resistivity is not a thermodynamic quantity, and, therefore, scaling is not expected on the basis of our assumption about the electronic free energy. Scaling is only inferred from the linear relationship between $\mathrm{A}$ and $\gamma^{2}$ and at the most then we should expect single-energy scaling only over that temperature range where the resistivity varies quadratically with temperature. This appears to be the case in UPt3 for which scaling the temperature axis, as for $\mathrm{CeSn} 3$, results in a universal curve $^{20}$ only at the lowest temperatures where $\rho \propto T^{2}$. See Fig. 5. Scaling to higher temperatures is possible if phonon contributions are at least qualitatively taken into account. ${ }^{21}$

Several examples indicate that resistivity scaling can be found over a much wider temperature range. Figure 6(a) gives the temperature-dependent resistance of $\mathrm{CeCu}_{6}$ at pressures between 1 bar and 17.4 kbar. ${ }^{22}$ The pronounced maximum in $\rho(T)$, near $T_{m}=15 \mathrm{~K}$ at atmospheric pressure, is common to several heavy-fermion materials having very large Sommerfeld coefficients and is believed ${ }^{10}$ to represent the cross-over from the high temperature regime where the scattering is dominated by non-interacting Kondo impurities to a low temperature regime in which the scattering becomes more coherent from site-to-site. At still higher pressures, Fig. 6(b), there is a qualitative change in the shape of $\rho(T)$ that most likely reflects a change in groundstate degeneracy, from $\mathrm{N}_{\mathrm{f}}=2$ at 1 bar to $\mathrm{N}_{\mathrm{f}}=6$ at the highest pressures, as $\mathrm{T}_{\mathrm{m}}$ exceeds the crystal-field splitting between the ground and first excited level which at 1 bar is only $65 \mathrm{~K}$. (It should be noted that there is also a strongly pressure dependent orthorhombic-to-monoclinic structural transition that complicates this interpretation but this is probably a minor perturbation since the transition does not manifest itself even in the 1-bar data.) At $122 \mathrm{kbar}$, the resistivity of $\mathrm{CeCu}_{6}$ resembles that of $\mathrm{CeSn}_{3}$ (Fig. 3(a)) and the $\mathrm{T}^{2}$-coefficients of resistivity are quite comparable, which supports the change in degeneracy interpretation. To demonstrate scaling for the data of Fig. 6, the resistance is divided by its value at $T_{m}$ and plotted versus $T / T_{m}$. This collapses the data onto a single curve for $0.1 \mathrm{~T}_{\mathrm{m}} \leq \mathrm{T} \leq 4 \mathrm{~T}_{\mathrm{m}}$. As found by Yomo et al. ${ }^{23}$, scaling at high temperatures begins to break down with increasing pressure and, further, the data of Fig. 6(b) fall onto two distinct curves, one for $\mathrm{P} \leq 57 \mathrm{kbar}$ and another for higher pressures as shown in Fig. 7. The loss of high temperature scaling at intermediate pressures may be due to a mixture of scattering processes, one intrinsic to the development of heavy quasiparticles (i.e. Kondo and RKKY interactions) and another reflecting the role of crystalline electric fields. That two separate scaling curves appear, with a clear break occurring between 57 and 73 kbar where there is also distinct change in $\rho(T)$, implies that the functional form of the scaling depends on groundstate degeneracy.

Although there are no other clear examples of this behavior, scaling of the pressuredependent resistivity in the form $R / R_{m}$ vs. $T / T_{m}$ has been observed ${ }^{13}$ in several other heavyfermion compounds, such as $\mathrm{UBe}_{13}, \mathrm{YbAgCu}_{4}, \mathrm{CeCu}_{2} \mathrm{Si}_{2}$ (provided crystal-field effects are considered), $\mathrm{URu}_{2} \mathrm{Si}_{2}$, and possibly $\mathrm{YbCu}_{2} \mathrm{Si}_{2}$ and $\mathrm{YbRh}_{2} \mathrm{Si}_{2}$. Given the rather broad spectrum 
of materials and associated heavy-fermion behavior for which resistance scaling is found (compounds with Sommerfeld coefficients ranging from 50 to $1600 \mathrm{~mJ} / \mathrm{mole} \mathrm{K}^{2}$, with degeneracy $\mathrm{N}=2$ as well as mixed-valent compounds with $\mathrm{N}=6$ or $8, \mathrm{Ce}$ and $\mathrm{U}$ compounds where the characteristic temperature, as measured by $\mathrm{T}_{\mathrm{m}}$ or $1 / \sqrt{\mathrm{A}}$, increases with pressure and $\mathrm{Yb}$ compounds where it decreases with pressure), it appears to be a very general characteristic. However, whether single-energy scaling is valid for $T<T_{\text {coh }}$ as well as $T>T_{\text {coh }}$ is less clear since scaling is typically determined over limited temperature or pressure ranges. To some extent this reflects inattention to experimental details, i.e. accounting for phonon and/or crystal-field contributions, use of non-isobaric and non-hydrostatic conditions, etc. A further complication arises from a significant anisotropy in transport properties found in some non-cubic heavy-fermion materials that may be sensitive to volume changes, but that has not been considered in any of the analyses.

Anisotropy is not a factor in analyzing pressure-dependent specific heat. In addition, for heavy-fermions phonon and crystal-field effects are generally insignificant below a few Kelvin. Therefore, if our assumption about the free energy scaling is correct, we expect that the pressuredependent specific heat should scale as $C_{p}$ versus $T / T_{0}(P)$, where $T_{0}$ is inversely proportional to the linear coefficient $\gamma$ of specific heat. The low temperature specific heat of $\mathrm{CeCu}_{6}$ at various pressures ${ }^{24}$ in plotted in Fig. 8(a). The coefficient $\gamma$ is strongly pressure dependent, decreasing from $1.67 \mathrm{~J} / \mathrm{mole}^{2}$ at ambient pressure to one-half this value at $8.8 \mathrm{kbar}$. These data are replotted in Fig. 8(b) where now the temperature axis has been multiplied by the linear specific heat coefficient appropriate to each applied pressure. These data scale very nicely up to a value of $\mathrm{T} \cdot \gamma$ $\approx 1$, which corresponds $0.6 \mathrm{~K}$ at ambient pressure and $1.2 \mathrm{~K}$ at $8.8 \mathrm{kbar}$. This temperature range includes and exceeds that over which $\rho \propto \mathrm{T}^{2}$. The lack of scaling above $\mathrm{T} \cdot \gamma \approx 1$ cannot be attributed to phonon contributions because at these temperatures the specific heat of $\mathrm{CeCu}_{6}$ far exceeds that of LaCu6. Given the large crystal-field splitting $(65 \mathrm{~K})$, it also seems unlikely that deviations from scaling represent a crystal-field effect. Hence, it may reflect a fundamental departure from single-energy scaling. The region over which all the data collapse onto a single curve reflects the fact that the temperature interval where $\mathrm{C} \propto \mathrm{T}$ increases with pressure at the same rate $\gamma$ decreases with pressure. That is, if $\gamma(\mathrm{P}) \propto 1 / \mathrm{T}_{0}(\mathrm{P})$, then $\mathrm{T}_{\mathrm{O}}(\mathrm{P})$ also sets the range over which $\mathrm{C} \propto \mathrm{T}$.

\section{COMPARISON BETWEEN EXPERIMENTS AND GRUNEISEN PARAMETERS}

Strictly speaking, the hypothesis that the free energy scales as $F_{e}=-N k_{B} T f\left(T / T_{O}\right)$ will lead to relations only between $\beta_{\mathrm{e}}, \mathrm{C}_{\mathrm{p}}$ and $\mathrm{BT}$ and hence equality of $\Omega_{\mathrm{e}}, \Omega_{\mathrm{c}}$ and $\Omega_{\beta}$. By extending the hypothesis to include magnetic field $\left(\mathrm{F}_{\mathrm{e}}=-\mathrm{NkBTf}\left(\mathrm{T} / \mathrm{T}_{\mathrm{O}}, \mathrm{H} / \mathrm{H}_{\mathrm{O}}\right)\right.$ ) then equality of $\Omega_{\chi}$ to $\Omega_{\mathrm{e}}$ will follow if $\mu \mathrm{H}_{\mathrm{o}}(\mathrm{V}) \propto \mathrm{kT}_{\mathrm{o}}(\mathrm{V})$. This is valid for the Kondo impurity problem and seems to be valid 
for most heavy-fermions as well. ${ }^{13}$ Scaling of the resistivity, however, does not follow from this assumption, again because it is not strictly a thermodynamic quantity.

We have seen that the resistivity of $\mathrm{CeCu}_{6}$ scales as $\mathrm{T} / \mathrm{T}_{\mathrm{m}}$ where $\mathrm{T}_{\mathrm{m}}$ is the temperature of the resistivity maximum. Frequently it is assumed that $T_{m}$ is proportional to $T_{0}$, where $T_{0}$ is determined from the low temperature specific heat through $\mathrm{T}_{0} \propto 1 / \gamma$. From these arguments, we expect $\gamma \propto 1 / \mathrm{T}_{\mathrm{m}}$. For $\mathrm{CeCu}_{6}$ an approximately linear relationship is found ${ }^{25}$ (Fig. 9) with logarithmic derivative $\partial \ell \mathrm{n} \gamma / \partial \ell \mathrm{n}\left(1 / \mathrm{T}_{\mathrm{m}}\right)=0.99$. That this derivative is essentially unity implies $\gamma=$ constant $/ T_{m}$, with intercept equal to 0 at $T_{m}=\infty$. (A logarithmic derivative identically unity is expected for a Kondo impurity for which $\gamma=\left(\mathrm{N}_{\mathrm{f}}-1\right) \pi / 6 \mathrm{~T}_{\mathrm{K}}$.) For $\mathrm{CeCu}_{6}$ the relationship $\mathrm{T}_{0} \propto$ $\mathrm{T}_{\mathrm{m}}$ appears to be valid, but for other materials, this logarithmic derivative differs substantially from unity: it is 0.7 for $\mathrm{UBe}_{13}$ and 1.9 for $\mathrm{YbCu}_{4} .5$, which suggests that $\mathrm{T}_{\mathrm{m}}$ and $\mathrm{T}_{\mathrm{O}}=1 / \gamma$ are not truly linearly related in these compounds. ${ }^{13}$ (The derivative formally should equal the ratio $\Omega_{\mathrm{C}} / \Omega \mathrm{T}_{\mathrm{R}}$, where $\Omega_{\mathrm{T}_{\mathrm{R}}}$ is defined below. As can be seen from Table $\mathrm{I}$, the value of this ratio is 1.04, 0.61 and 2.09 for $\mathrm{CeCu}_{6}, \mathrm{UBe}_{13}$ and $\mathrm{YbCu}_{4} .5$, respectively.) A second assumption concerning the resistivity that can be examined from high pressure data is whether $\gamma(P)$ is proportional to $\sqrt{A}(\mathrm{P})$. Taking the observation of Kadowaki and Woods ${ }^{6}$ that $A=c \gamma^{2}+b$, where $c$ and $b$ are constants independent of material, leads to $\partial \ell n \gamma / \partial \ell n \sqrt{A}=1+b / c \gamma^{2}$. That is, larger $\gamma$ values should give a logarithmic derivative closer to unity. From plots of $\gamma(\mathrm{P})$ vs. $\sqrt{\mathrm{A}}(\mathrm{P})$ for $\mathrm{UPt}_{3}\left(\gamma \approx 450 \mathrm{~mJ} / \mathrm{mole} \mathrm{K}^{2}\right)$ and $\mathrm{UBe}_{13}\left(\gamma \approx 1000 \mathrm{~mJ} / \mathrm{mole}^{2}\right)$ we find ${ }^{13}$ this derivative to be 1.2 and 2.7, respectively. (This derivative should equal $\Omega_{\mathrm{C}} / \Omega_{\mathrm{A}}$, which from Table $\mathrm{I}$ is 1.0 and 3.7 in the two cases.) For UPt3 the value is essentially unity, but for $\mathrm{UBe}_{13}$ it is not and suggests that $\gamma$ and $\sqrt{\mathrm{A}}$ are not simply related in $\mathrm{UBe} 13$. From considering relationships among these characteristic scales, it is clear that caution must be taken in equating them, which often has not been the case.

As discussed in the Introduction, a straightforward test of the scaling assumption for the electronic free energy is to examine the relationship between Gruneisen parameters. Table I gives a compilation of Gruneisen parameters for heavy-fermion compounds having a wide range of Sommerfeld coefficients. In addition to Gruneisen parameters mentioned in the Introduction, Table I includes $\Omega_{\mathrm{T}}=-\partial \ell \mathrm{n} \mathrm{T}_{\mathrm{m}} / \partial \ell_{\mathrm{nV}}$, where $\mathrm{T}_{\mathrm{m}}$ is the temperature at which the susceptibility is a maximum and $\Omega \mathrm{T}_{\mathrm{R}}=-\partial \ell \mathrm{n} \mathrm{T}_{\mathrm{R}} / \partial \ell \mathrm{nV}$, where $\mathrm{T}_{\mathrm{R}}$ is either the temperature at which the resistivity is a maximum or, if there is no resistance maximum, the temperature where $\partial^{2} \rho / \partial T^{2}$ changes sign. There are several points to make about the entries in this table. (1) Gruneisen parameters of Ceand U-based compounds are positive, signifying an increase in the characteristic energy scale with pressure, whereas just the opposite behavior is found for those compounds based on $\mathrm{Yb}$. (2) Except for $\mathrm{UBe}_{13}$ and $\mathrm{CeCu}_{2} \mathrm{Si}_{2}$, Gruneisen parameters for a given material do not vary by more than $\pm 50 \%$ from the average even though some parameters are determined at very low temperatures and others at one-to-two orders of magnitude higher temperatures. In some cases, e.g. $\mathrm{CeCu}_{6}$, 
$\mathrm{CeRu}_{2} \mathrm{Si}_{2}, \mathrm{UAl}_{2}$ and $\mathrm{UPt} 3$, agreement is substantially better than $\pm 50 \%$ and within experimental variation from measurement to measurement. (3) Except for $\mathrm{CeRu}_{2} \mathrm{Si}_{2}$, there is a general trend for the absolute value of Gruneisen parameters to increase with increasing electronic specific heat coefficient $\gamma$.

There are several sources of uncertainty in the absolute values of $\Omega$. In part, some of the variation may be caused by use of different samples for the different measurements. In addition, $\Omega_{c}$ and $\Omega_{e}$ are true volume measurements; whereas, $\Omega_{\chi}$ could be affected by crystallographic anisotropy in non-cubic materials (as could $\Omega_{\mathrm{T}}, \Omega_{\mathrm{A}}$ and $\Omega_{\mathrm{T}}$ ). A further point to consider is that, in calculating electronic contributions to the volume-thermal expansion coefficient and specific heat, background corrections should be applied. For large- $\gamma$ materials, these corrections are generally small but for others background subtraction can introduce greater uncertainty in the absolute value of $\Omega_{e}$. Finally, Gruneisen parameters can be strongly temperature dependent, ${ }^{26}$ as shown for $\mathrm{CeCu}_{6}$ in Fig. 10. Strictly speaking, $\Omega_{\mathrm{c}}, \Omega_{\chi}$ and $\Omega_{\mathrm{e}}$ should be determined in the $\mathrm{T} \rightarrow 0, \mathrm{P} \rightarrow 0$ limit and compared to determinations of $\Omega_{\mathrm{T}}$ and $\Omega_{\mathrm{T}}$, which are measured at higher temperature. For the values included in Table I we have tried to use the lowest temperature data available and have attempted to evaluate pressure derivatives in the $\mathrm{P} \rightarrow 0$ limit. Even with these precautions, the listings in Table I must be viewed with some caution.

There are several cases that deserve further comment and we begin with CeSn3. As noted above, scaling of the susceptibility appears valid over an interval $30<\mathrm{T}<300 \mathrm{~K}$. This is corroborated by the near-equality of $\Omega_{\chi}$ and $\Omega_{\mathrm{T}}$ with $\Omega_{\mathrm{e}}=10$, determined by comparing $\mathrm{C}_{\mathrm{p}}$ and $\beta_{\mathrm{e}}$ over the temperature range $4-300 \mathrm{~K}$. These results suggest single-energy scaling is valid both at moderately high temperatures, where the electronic system behaves as non-interacting Kondo impurities, and at low temperatures where the $4 \mathrm{f}$ lattice is becoming coherent. This may reflect the absence of strong antiferromagnetic correlations in the groundstate of this mixed-valence compound. The resistivity also displays $\mathrm{T} / \mathrm{T}_{\mathrm{O}}$ scaling for $20<\mathrm{T}<50 \mathrm{~K}$, with $\Omega \mathrm{T}_{\mathrm{R}}$ within $30 \%$ of the average value for $\Omega$. However, below $20 \mathrm{~K}$ the resistivity scaling breaks down and $\rho$ varies as $\mathrm{T}^{3}$. Recalling that the susceptibility scaling is uncertain at low $\mathrm{T}$ due to the background subtraction, we suggest that whether single-energy scaling is truly valid at low temperatures warrants further investigation.

$\mathrm{CeCu}_{6}$ also appears to exhibit single-energy scaling. The resistivity for $4<\mathrm{T}<150 \mathrm{~K}$ can be scaled onto a single curve as a funciion of $\mathrm{T} / \mathrm{T}_{\mathrm{m}}(\mathrm{P})$ for all $\mathrm{P} \leq 50 \mathrm{kbar}$ and the associated parameter $\Omega_{\mathrm{T}}=70$ agrees well with the value $\Omega_{\mathrm{C}}=73$ determined from the low temperature $(0.1<\mathrm{T}<1 \mathrm{~K}) \mathrm{T}$-linear specific heat. The parameter $\Omega_{\mathrm{e}}$ also has a value $\approx 80$ near $0.2 \mathrm{~K}$. (Fig. 10) Interestingly, single-energy scaling of the thermodynamics and transport occurs in the presence of two energy scales in the neutron scattering, ${ }^{17}$ a single-ion Kondo energy $\mathrm{T}_{\mathrm{K}}=$ $5 \mathrm{~K}$ and a scale of $2.5 \mathrm{~K}$ below which antiferromagnetic correlations develop. These correlations saturate below $1 \mathrm{~K}$, where the specific heat becomes linear in temperature. In $\mathrm{UPt}^{27}$ and 
$\mathrm{CeRu}_{2} \mathrm{Si}_{2}{ }^{17}$, antiferromagnetic correlations between $\mathrm{f}$-moments also coexist with Kondo-like spin fluctuations, each with their own characteristic energy scale. In spite of these two clearly defined scales, single-energy scaling is indicated by the near equality of low temperature Gruneisen parameters $\Omega_{\mathrm{c}}, \Omega_{\mathrm{A}}$ and $\Omega_{\mathrm{e}}$ with the higher temperature parameter $\Omega_{\mathrm{T}}$, which is associated with the proximity to a metamagnetic transition at a field $\mu \mathrm{H}_{\mathrm{o}} \approx \mathrm{kB}_{\mathrm{B}}$. Also like CeCu6. antiferromagnetic correlations in $\mathrm{CeRu}_{2} \mathrm{Si}_{2}$ saturate at a temperature not far above that where $\mathrm{C}_{\mathrm{p}}$ is linear-in- $\mathrm{T}$; however, in both cases $\rho$ is quadratic in temperature only at much lower temperatures.

However, in other materials there are interesting apparent exceptions to single-energy scaling. CePd 3 exhibits scaling of the resistivity above $50 \mathrm{~K}$, but $\rho$ is nearly independent of pressure at lower temperatures. ${ }^{28}$ This is reflected in the inequality of $\Omega_{\mathrm{A}}$ and $\Omega \mathrm{T}_{\mathrm{R}}$. There is substantial variation among Gruneisen parameters for $\mathrm{UBe}_{13}$. Even the two thermodynamic parameters $\Omega_{c}$ and $\Omega_{\chi}$ differ by a factor of seven. One interpretation of this is that the Wilson ratio increases with pressure, which implies that $\mathrm{UBe}_{13}$ would be pushed toward a magnetic instability at high pressures. Indeed, high pressure thermoelectric power measurements ${ }^{29}$ have been interpreted as showing evidence for a magnetic transition at pressures near $67 \mathrm{kbar}$, but this is not reflected in the electrical resistance ${ }^{30}$ and remains an open question. In addition, $\Omega_{\mathrm{A}}$ is much smaller than the electronic parameters which, as mentioned above, reflects the non-linear relation between $\sqrt{\mathrm{A}}$ and $\gamma$. Finally, we emphasize that $\mathrm{CeRu}_{2} \mathrm{Si}_{2}$ has an anomalously large value of $\Omega$ relative to $\gamma$ and is a marked exception to the general trend among other compounds that $\Omega \propto \gamma$. We suggest that the existence of the low field $(8 \mathrm{~T})$ metamagnetic transition in $\mathrm{CeRu}_{2} \mathrm{Si}_{2}$ places it in proximity to a $\mathrm{T}=0$ fixed point, which may dominate the scaling ${ }^{31}$ and explain the anomalously large Gruneisen parameters.

\section{SUMMARY}

We have considered consequences of assuming that the electronic free energy of heavyfermion materials is a universal function of a volume-dependent energy scale $T_{O}$ which arises from the interplay between Kondo and RKKY interactions. This basic assumption predicts that thermodynamically derived quantities should scale as $T / T_{O}(V)$ and that Gruneisen parameters for these quantities should be equal. Further, from the observation that a variety of heavy-fermion compounds at atmospheric pressure satisfy $\mathrm{A} \propto \gamma^{2}$, single-energy scaling may apply as well to the low temperature resistivity at temperatures where $\rho \propto \mathrm{T}^{2}$. Scaling the volume-dependent magnetic susceptibility and electrical resistivity as $\mathrm{T} / \mathrm{T}_{\mathrm{O}}(\mathrm{V})$ does indeed produce a single curve over broad ranges of temperatures and pressures for several heavy-fermion materials. However, similar scaling of the specific heat holds, at least in $\mathrm{CeCu}_{6}$, for temperatures only between $60 \mathrm{mK}$ and about $1 \mathrm{~K}$; the significance of deviations from scaling above $1 \mathrm{~K}$ is unclear. The electronic specific heat coefficient is a linear function of $1 / \mathrm{T}_{\mathrm{m}}(\mathrm{P})$ and $\sqrt{\mathrm{A}}(\mathrm{P})$ for several materials. For $\mathrm{CeCu}_{6}$. $\partial \ell n \gamma / \partial \ell n\left(1 / T_{m}\right)$ is essentially unity. If $T_{m}$ is proportional to the single-ion Kondo energy $T_{K}$, 
then $\gamma$ is proportional to $1 / \mathrm{T}_{\mathrm{K}}$ which is somewhat surprising since $\gamma$ is measured in a temperature range where antiferromagnetic correlations are clearly observed in neutron scattering. In other

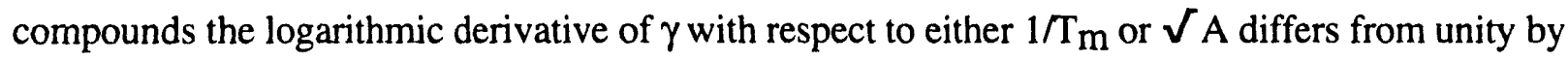
factors of two to three, indicating that in these the pressure dependences of $1 / \sqrt{\mathrm{A}}$ and $\mathrm{T}_{\mathrm{m}}$ are not simply proportional to $T_{O}(P)$. Agreement among Gruneisen parameters, deduced from the volume dependence of the magnetic susceptibility, electronic specific heat and electrical resistivity, is in several cases good (e.g. $\mathrm{CeCu}_{6}, \mathrm{CeSn}_{3}, \mathrm{CeRu}_{2} \mathrm{Si}_{2}, \mathrm{UAl}_{2}$ and $\mathrm{UPt} 3$ ). In other compounds there appear to be significant deviations between low temperature parameters $\left(\Omega_{c}, \Omega_{\chi}\right.$ and $\left.\Omega_{A}\right)$ and high temperature parameters $\left(\Omega_{\mathrm{T}}\right.$ and $\left.\Omega_{\mathrm{T}_{\mathrm{R}}}\right)$. For UBe $13, \Omega \chi$ is much less than $\Omega_{\mathrm{c}}$, which may indicate an approach to a magnetic instability at high pressure. $\mathrm{CeCu}_{6}$ is an interesting example where Gruneisen parameters determined at very low temperatures, i.e. $\Omega_{\mathrm{c}}$ and $\Omega_{\mathrm{e}}$, agree with $\Omega_{T_{R}}$ determined at much higher temperatures, yet $\Omega_{c}$ and $\Omega_{e}$ are strongly temperature dependent. Indeed, the free energy scaling assumption predicts a temperature independent Gruneisen parameter $\Omega_{e}$ but this constancy has not been observed for a single material; nevertheless, agreement among Gruneisen parameters deduced at substantially different temperatures is often good.

These observations present a confusing picture. It seems that, if single-energy scaling is a valid concept, the electronic system in some undefined way senses the presence of both Kondo and RKKY interactions over wide temperature and pressure ranges such that it mimics a single-energy scale which becomes increasingly well-defined at low temperatures. From one perspective, this interpretation could be viewed as a restatement of the fundamental heavy-fermion problem: namely, how are high temperature, local moment degrees of freedom transformed into a band of strongly renormalized quasiparticles at low temperature? From another, it is a stronger statement, namely, that this transformation proceeds in a specific way such that the relationship between intraand intersite interactions produces a unique energy scale at $T=0$. Alternatively, the antiferromagnetic correlations responsible for coherence may in some cases have only a marginal effect on the quantities $C_{p}$, and $\chi$, so that although coherence is present, the thermodynamics is dominated by single-ion Kondo behavior. Though experiments provide some evidence for free energy scaling, none of the specific issues considered, i.e. validity of scaling physical properties and equality of various Gruneisen parameters, has been established unambiguously. Given the importance of these issues, additional theoretical and experimental work clearly is needed.

Experimental short-comings have been mentioned throughout and these need to be addressed. On the theoretical side, very little has been done to calculate the temperature or volume dependence of the local moment-conduction electron hybridization and of the f-occupation number (which has not been discussed at all but which could be important). How intersite (RKKY) and intrasite (Kondo) interactions produce a band of heavy quasiparticles at low temperatures, characterized presumably by a single-energy scale, is, of course, an outstanding question that deserves attention. 


\section{ACKNOWLEDGMENTS}

Work at Los Alamos was performed under the auspices of the U.S. Department of Energy.

JML acknowledges support of the Center for Materials Science, through the UC Personnel Assignment Program, and of the UC/Los Alamos INCOR program. 


\section{Figure Captions}

Fig. 1. (a) $4 \mathrm{f}$ contribution to the magnetic susceptibility of $\mathrm{CeSn} 3$ at several pressures plotted as a function of temperature. The 1-bar susceptibility of $\mathrm{LaSn}_{3}$ has been subtracted from these data and assumed to be pressure independent. Data are from Ref. 11.

(b) Data of (a), normalized to the value of $\chi$ at $T_{m}$, where $T_{m}$ is the temperature at which $\chi$ is a maximum as a function of reduced temperature $\mathrm{T} / \mathrm{T}_{\mathrm{m}}$.

Fig. 2. (a) Magnetic susceptibility of UPt3 obtained for a magnetic field of $5 \mathrm{~T}$ applied in the hexagonal basal plane. Data from Ref. 14.

(b) Data from (a) normalized to the maximum value of the susceptibility $\chi_{\mathrm{m}}$ versus $\mathrm{T} / \mathrm{T}_{\mathrm{m}}$

Fig. 3. (a) Temperature-dependent resistivity of $\mathrm{CeSn} 3$ at several pressures.

(b) Data of (a) plotted as a function of reduced temperature $T / T_{0}(P)$, where $T_{O}(P)$ is the

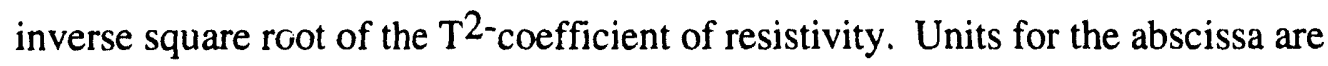
$(\mu \Omega \mathrm{cm})^{1 / 2}$.

Fig. 4. (a) Data of Fig. 3(a) plotted as $T / T_{0}(P)$ showing deviations from scaling at low temperatures. The residual resistivity $\rho_{0}$ has been subtracted.

(b) Resistivity as a function of $\mathrm{T}^{3}$ for $\mathrm{CeSn} 3$ at various pressures.

Fig. 5. (a) Resistivity of UPt3 at selected pressures versus temperature. Data from Ref. 20.

(b) Data of (a), plotted as a function of reduced temperature $T / T_{0}(P)$, where $T_{0}(P)$ is the inverse square root of the $\mathrm{T}^{2}$-coefficient of resistivity. Units for the abscissa are $(\mu \Omega \mathrm{cm})^{1 / 2} . T_{0}$ is a linear function of pressure over the entire pressure range with slope $\partial \mathrm{T}_{\mathrm{o}} / \partial \mathrm{P}=0.045\left(\mu \Omega \mathrm{cm} / \mathrm{K}^{2}\right)^{-1 / 2} / \mathrm{kbar}$. Note that scaling holds only over the range where $\rho \propto \mathrm{T}^{2}$.

Fig. 6 (a) Temperature-dependent resistance of $\mathrm{CeCu}_{6}$ at pressures to $17.4 \mathrm{kbar}$. Data from Ref. 22. 
(b) Selected resistance versus temperature curves for $\mathrm{CeCu}_{6}$ at pressures to $122 \mathrm{kbar}$. Data from Ref. 23. Note the qualitatively different temperature dependence that occurs between 41 and $73 \mathrm{kbar}$.

Fig. 7. Resistance of $\mathrm{CeCu}_{6}$ normalized to its maximum value at $\mathrm{T}_{\mathrm{m}}$ as a function of $\mathrm{T} / \mathrm{T}_{\mathrm{m}}$. Data from Ref. 23. The temperature-dependent resistivity of $\mathrm{LaCu}_{6}$ at 1 bar has been subtracted from each data set before normalization. The existence of two scaling curves most likely reflects a change in groundstate degeneracy.

Fig. 8. (a) Temperature-dependent specific heat of $\mathrm{CeCu}_{6}$ at various pressures. Data from Ref. 24. The T-linear specific heat $(\gamma)$ decreases from $1.67 \mathrm{~J} /$ nole $\mathrm{K}^{2}$ at 1 bar to about one half this value at the highest pressure.

(b) Specific heat data from (a) plotted as a function of $\mathrm{T} \gamma$.. Under the assumption that $\gamma \sim 1 / T_{0}$, these data should scale onto a single curve.

Fig. 9. Sommerfeld coefficient $\gamma$ as a function of $1 / \mathrm{T}_{\mathrm{m}}$ for $\mathrm{CeCu}$, where $\mathrm{T}_{\mathrm{m}}$ is the temperature at which the resistance is a maximum. Pressure is the implicit variable. The solid line gives a logarithmic derivative of $\gamma$ with respect to $1 / \mathrm{T}_{\mathrm{m}}$ of 0.99 .

Fig. 10. Temperature dependence of $\Omega_{c}$ (open circles) for $\mathrm{CeCu}_{6}$ calculated from the data in Fig. 8 (a) by fitting the values of $\mathrm{C}_{\mathrm{p}} / \mathrm{T}$ at four temperatures to a functional form $\left.\gamma=\gamma_{0}{ }^{(-\Omega P / B}\right)$ Variation in $\Omega_{\mathrm{e}}$ (open diamonds) for $\mathrm{CeCu}_{6}$ at low temperatures. Data from Ref. 26 


\section{REFERENCES}

1. N. Andrei, K. Fuyura and J.H. Lowenstein, Rev. Mod. Phys. 55, 331 (1983).

2. B. Lüthi, J. Magn. Magn. Mater. 52, 70 (1985).

3. S. Schilling, Adv. Phys. $\underline{28}, 657$ (1979).

4. Z. Fisk, D.W. Hess, C.J. Pethick, D. Pines, J.L. Smith, J.D. Thompson and J.O. Willis, Science 239, 33 (1988).

5. See for instance: P.A. Lee, T.M. Rice, J.W. Serene, L.J. Sham and J.W. Wilkins, Comments Conds. Mat. Phys. 12, 99, 1986.

6. K. Kadowaki and S.B. Woods, Solid State Commun. 표, 307 (1986).

7. N.E. Bickers, D.L. Cox and J.W. Wilkins, Phys. Rev. B ㅁ6, 2036 (1987).

8. J.M. Lawrence and D.L. Mills, Comments Conds. Mat. Phys. 15, 163 (1991).

9. S. Doniach, in: Valence Instabilities and Related Narrow Band Phenomena, ed. R.D. Parks (Plenum, New York, 1977) p. 169.

10. See for instance: N. Grewe and F. Steglich, in: Handbook on the Physics and Chemistry of Rare Earths, vol. 14, eds. K.A. Gschneidner and L. Eyring, (Elsevier, Amsterdam, 1991) p. 343.

11. S.A. Shaheen, J.S. Schilling, S.H. Liu and O.D. McMasters, Phys. Rev. B $\underline{27}, 4325$ (1983).

12. V.T. Rajan, Phys. Rev. Lett. 51, 308 (1983).

13. J.D. Thompson and J.M. Lawrence, in: Handbook on the Physics and Chemistry of Rare Earths and Actinides, Vol. 19, eds. K.A. Gschneidner, G. Lander, L. Eyring and G. Choppin (Elsevier, Amsterdam) in press.

14. A. de Visser, A. Menovsky and J.J.M. Franse, Physica B 147, 81 (1987).

15. P.H. Frings, J.J.M. Franse, F.R. de Boer and A. Menovsky, J. Magn. Magn. Mater. 31-34, 240 (1983).

16. P. Haen, J. Flouquet, F. Lapierre, P. Lejay and G. Remenyi, J. Low Temp. Phys. 67, 391 (1987).

17. J. Rossat-Mignod, L.P. Regnault, J.L. Jacoud, C. Vettier, P. Lejay, J. Flouquet, E. Walker, D. Jaccard and A. Amato, J. Magn. Magn. Mater. 76 \& 77, 376 (1988). 
18. J. Voiron, J-M. Mignot, P. Lejay, P. Haen and J. Flouquet, J. Phys. (France) 49, 1555 (1988).

19. J-M. Mignot, J. Flouquet, P. Haen, F. Lapierre, L. Peuch and J. Voiron, J. Magn. Magn. Mater. $76 \& 77,97$ (1988).

20. A. Ponchet, J-M. Mignot, A. de Visser, J.J.M. Franse and A. Menovsky, J. Magn. Magn. Mater. 54-57, 399 (1986).

21. M.S. Wire, J.D. Thompson and Z. Fisk, Phys. Rev. B 쓰, 5591 (1984).

22. J.D. Thompson and Z. Fisk, Phys. Rev. B 쓰, 5591 (1984).

23. S. Yomo, P.H. Hor, R.L. Meng, C.W. Chu and J. Sasaki, J. Magn. Magn. Mater. 76 \& 77, 257 (1988).

24. N.E. Phillips, R.A. Fisher, S.E. Lacy, C. Marcenat, J.A. Olson, J. Flouquet, A. Amato, D. Jaccard, Z. Fisk, A.L. Giorgi, J.L. Smith and G. R. Stewart, in: Theoretical and Experimental Aspects of Valence Fluctuations and Heavy Fermions, eds. L.C. Gupta and S.K. Malik (Plenum, New York, 1987) p. 141.

25. J. D. Thompson, Z. Fisk and G. Lonzarich, Physica B $\underline{161}, 317$ (1989).

26. A. de Visser, J.J.M. Franse, A. Lacerda, P. Haen and J. Flouquet, Physica B 163, 49 (1990).

27. G. Aeppli, E. Bucher, A.I. Goldman, G. Shirane, C. Broholm and J.K. Kjems, J. Magn. Magn. Mater. 76 \& 77,385 (1988).

28. J.M. Lawrence, J.D. Thompson and Y.Y. Chen, Phys. Rev. Lett. 54, 2537 (1985).

29. S.Y. Mao, D. Jaccard, J. Sierro, Z. Fisk and J.L. Smith, J. Magn. Magn. Mater. 76 \& 77, 241 (1988).

30. M.C. Aronson, J.D. Thompson, J.L. Smith, Z. Fisk and M.W. McElfresh, Phys. Rev. Lett. $\underline{63}, 2311$ (1989). 
31. M.A. Continentino, Phys. Rev. B 47, 11587 (1993). 
Table I. Summary of Gruneisen parameters for several Ce-, Yb- and U-based heavy-fermion compounds. Entries from Ref. 13. Definitions: $\gamma$-Sommerfeld coefficient at $1 \mathrm{bar} ; \Omega_{\mathrm{c}}=\partial \ell \mathrm{n} \gamma / \partial \ell \mathrm{nV} ; \Omega_{\chi}=\partial \ell \mathrm{n} \chi(0) / \partial \ell \mathrm{nV}$;

$\Omega_{\mathrm{T}}=-\partial \ell \mathrm{nT}_{\mathrm{m}} \chi / \partial \ell \mathrm{nV} ; \Omega_{\mathrm{A}}=-\partial \ell \mathrm{n}(1 / \sqrt{\mathrm{A}}) / \partial \ell \mathrm{nV} ; \Omega_{\mathrm{T}}=-\partial \ell \mathrm{nT}_{\mathrm{m}} \mathrm{e} / \partial \ell \mathrm{nV} ; \Omega_{\mathrm{e}}=\beta_{\mathrm{e}} \mathrm{VBT}_{\mathrm{T}} / \mathrm{C}_{\mathrm{p}} ;$ nd-not determined.

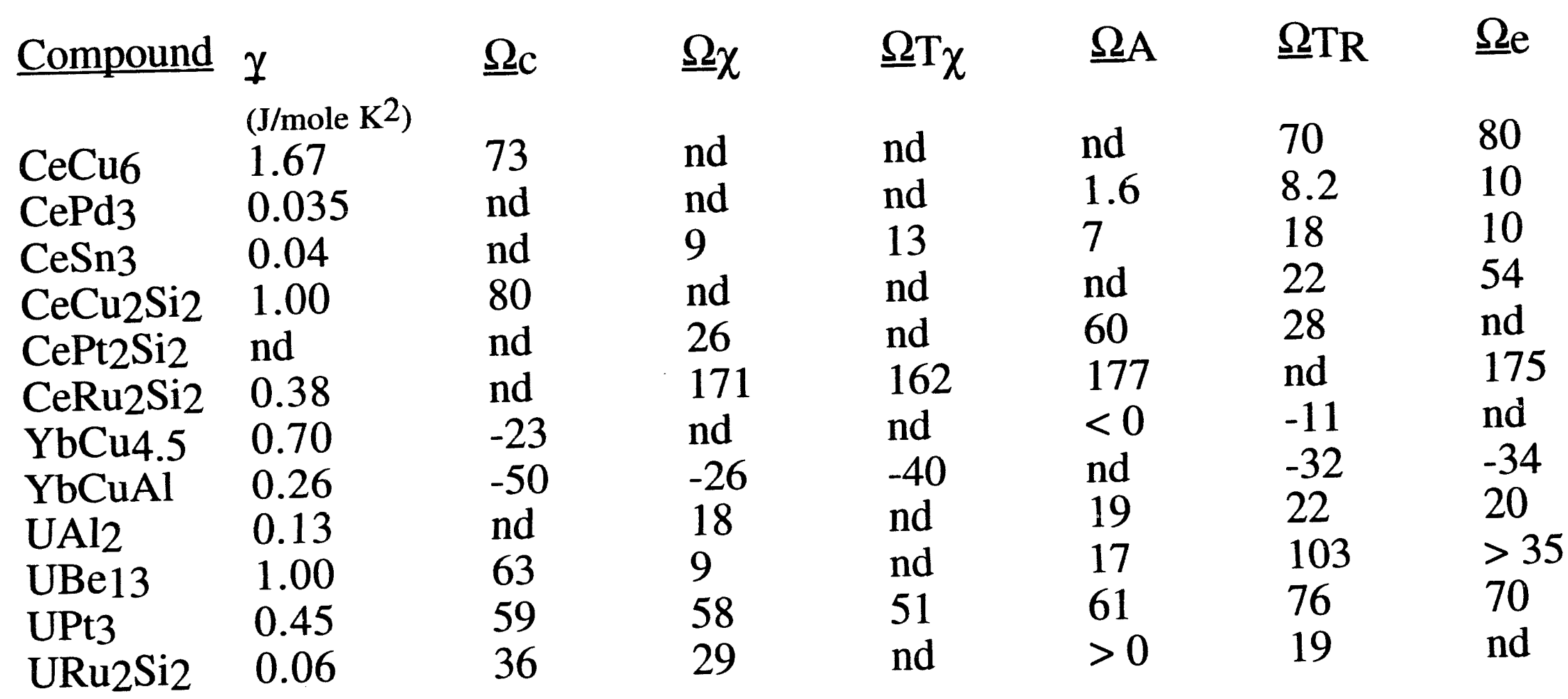




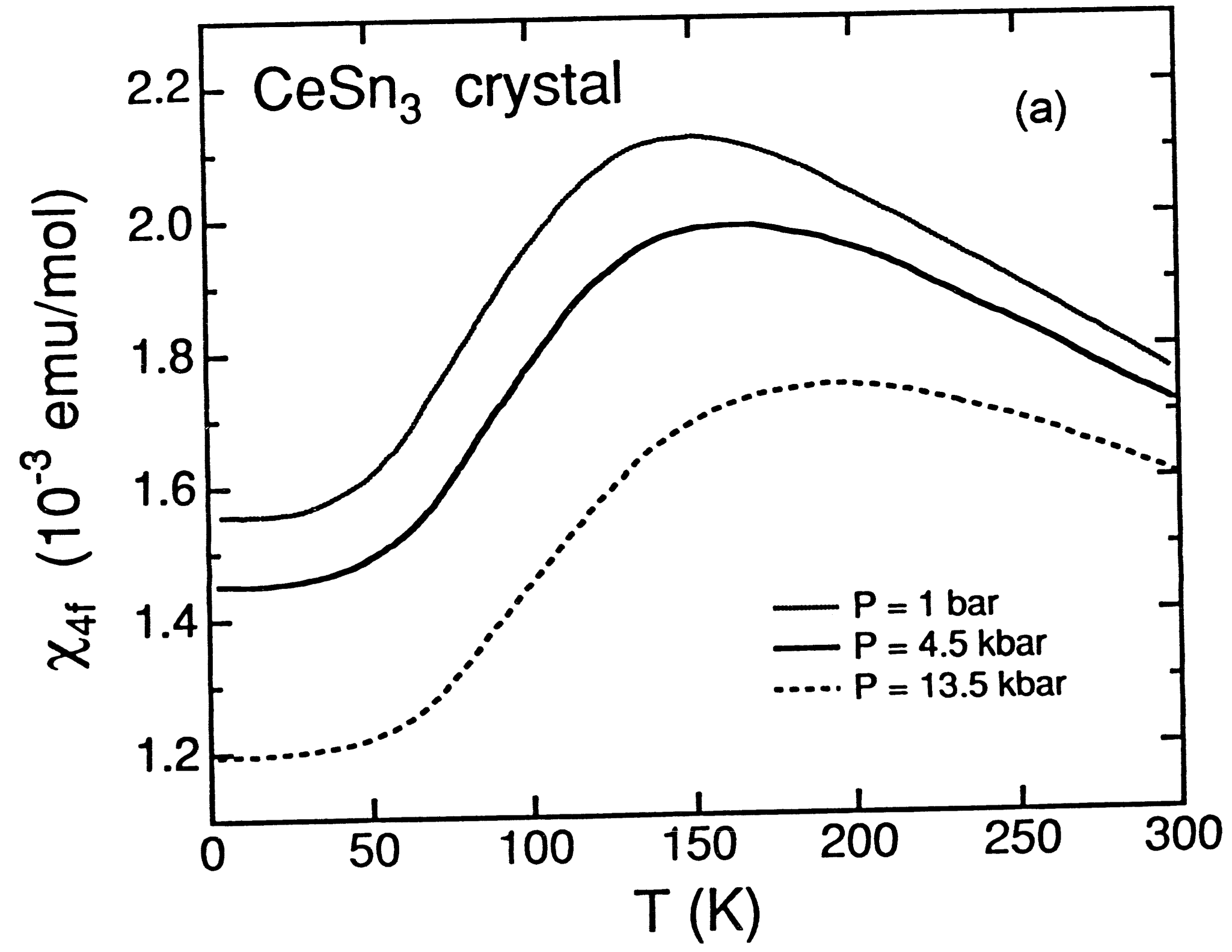




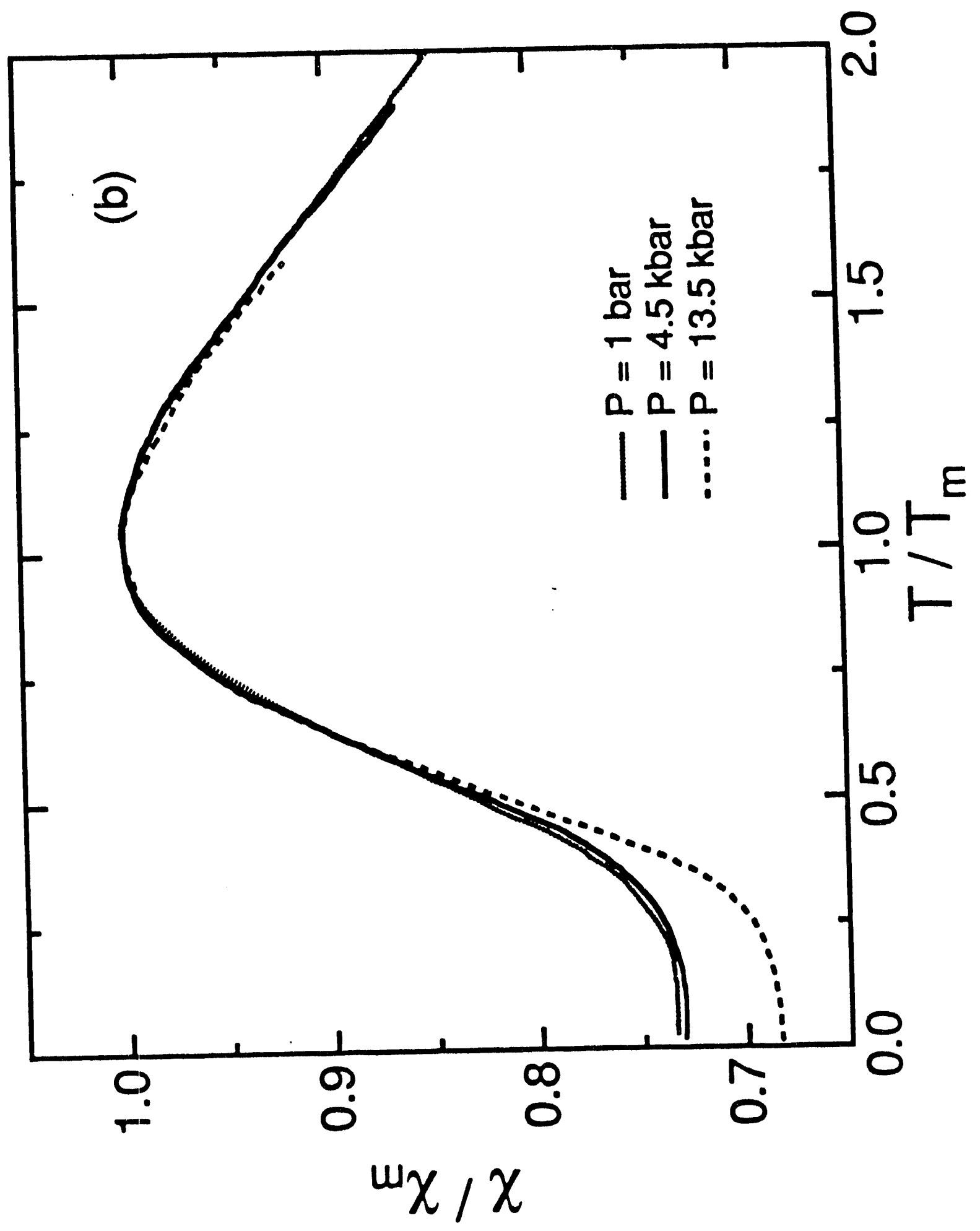




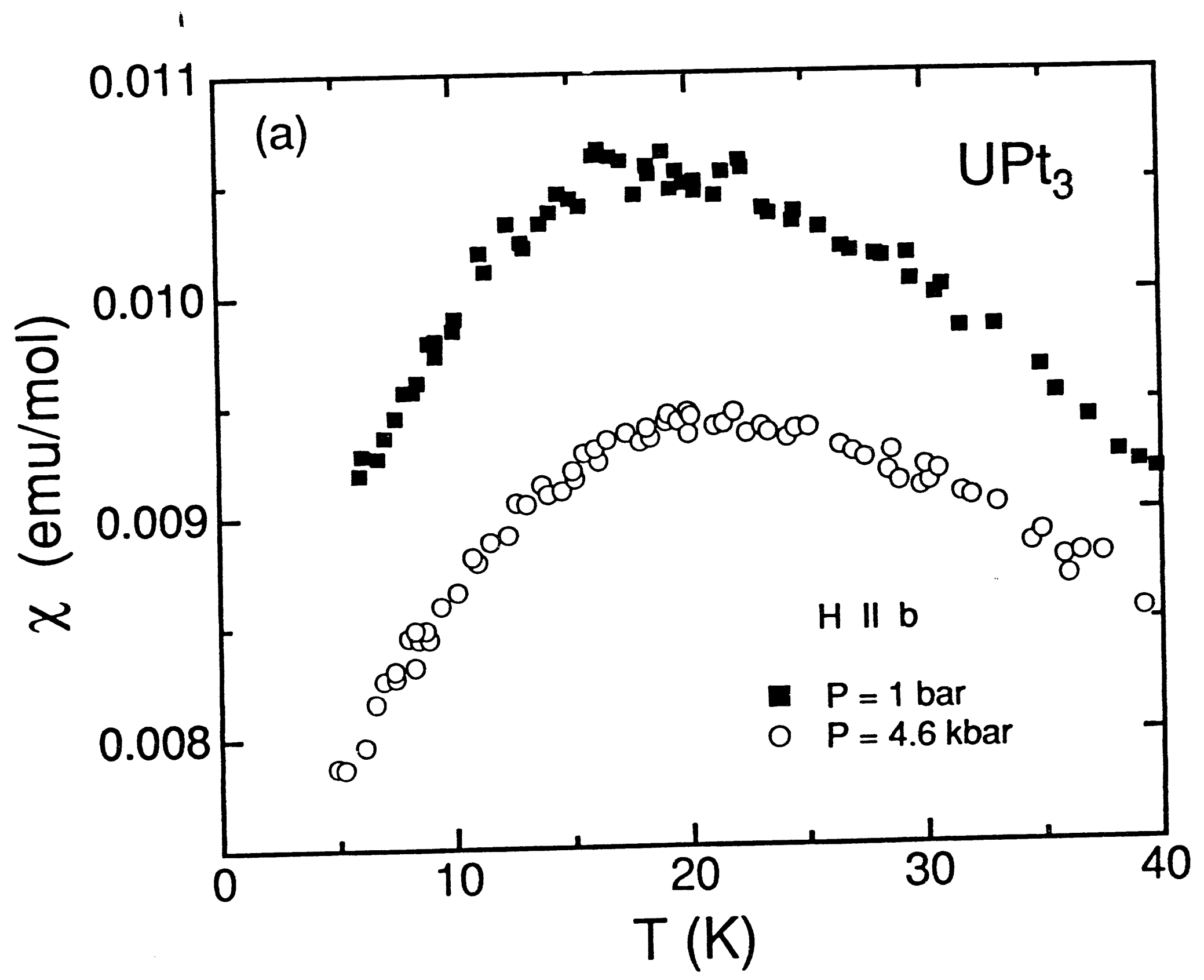




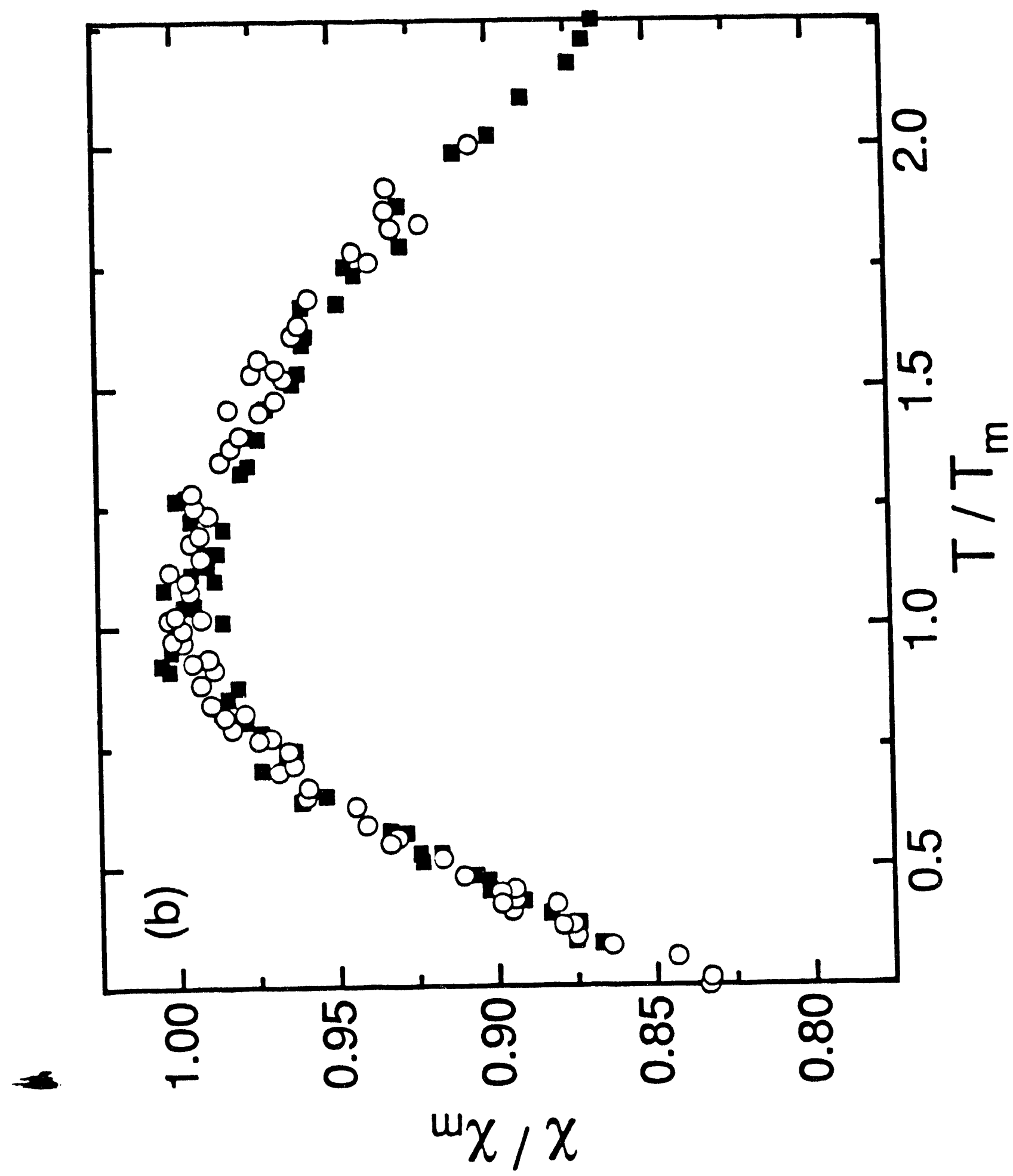




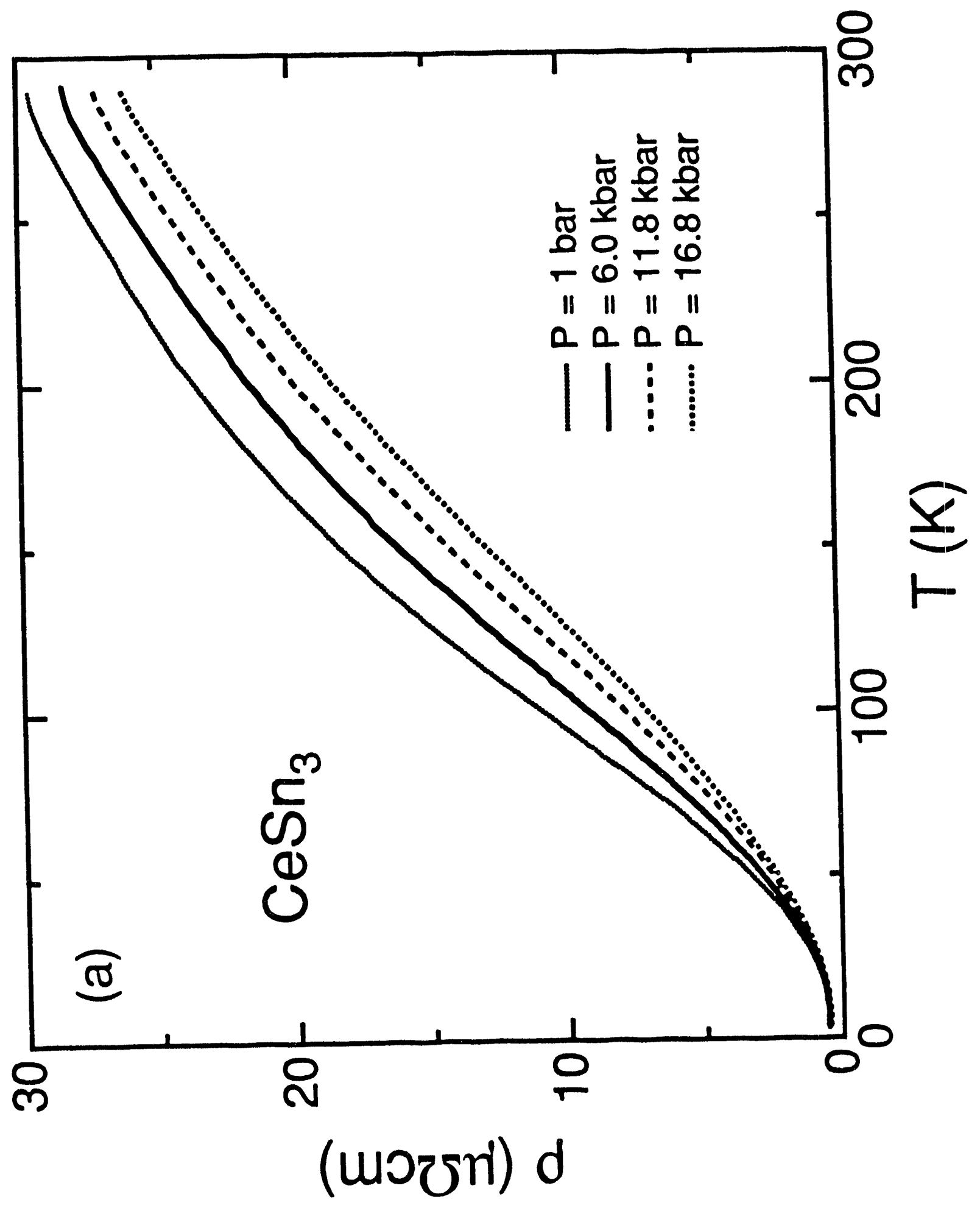




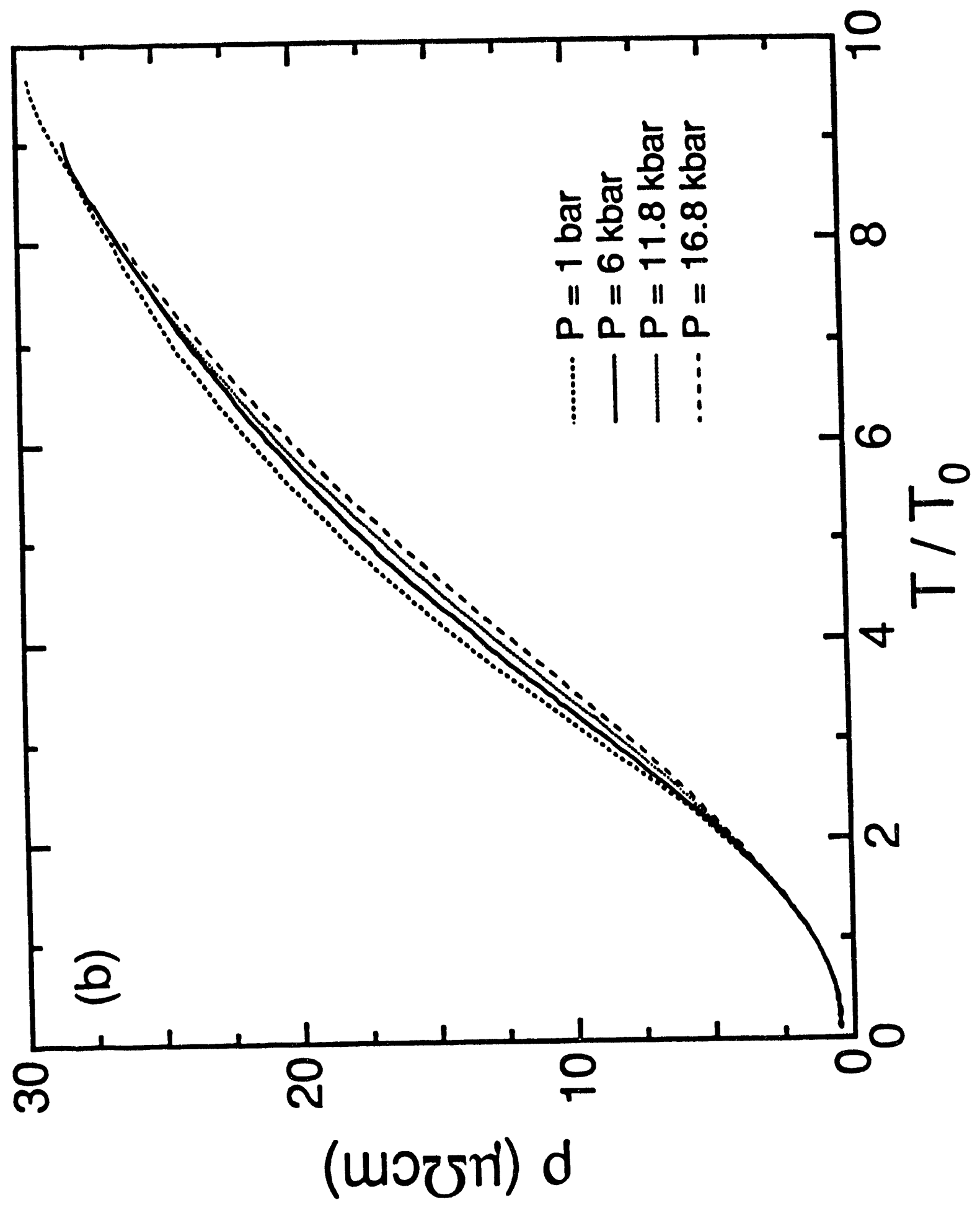




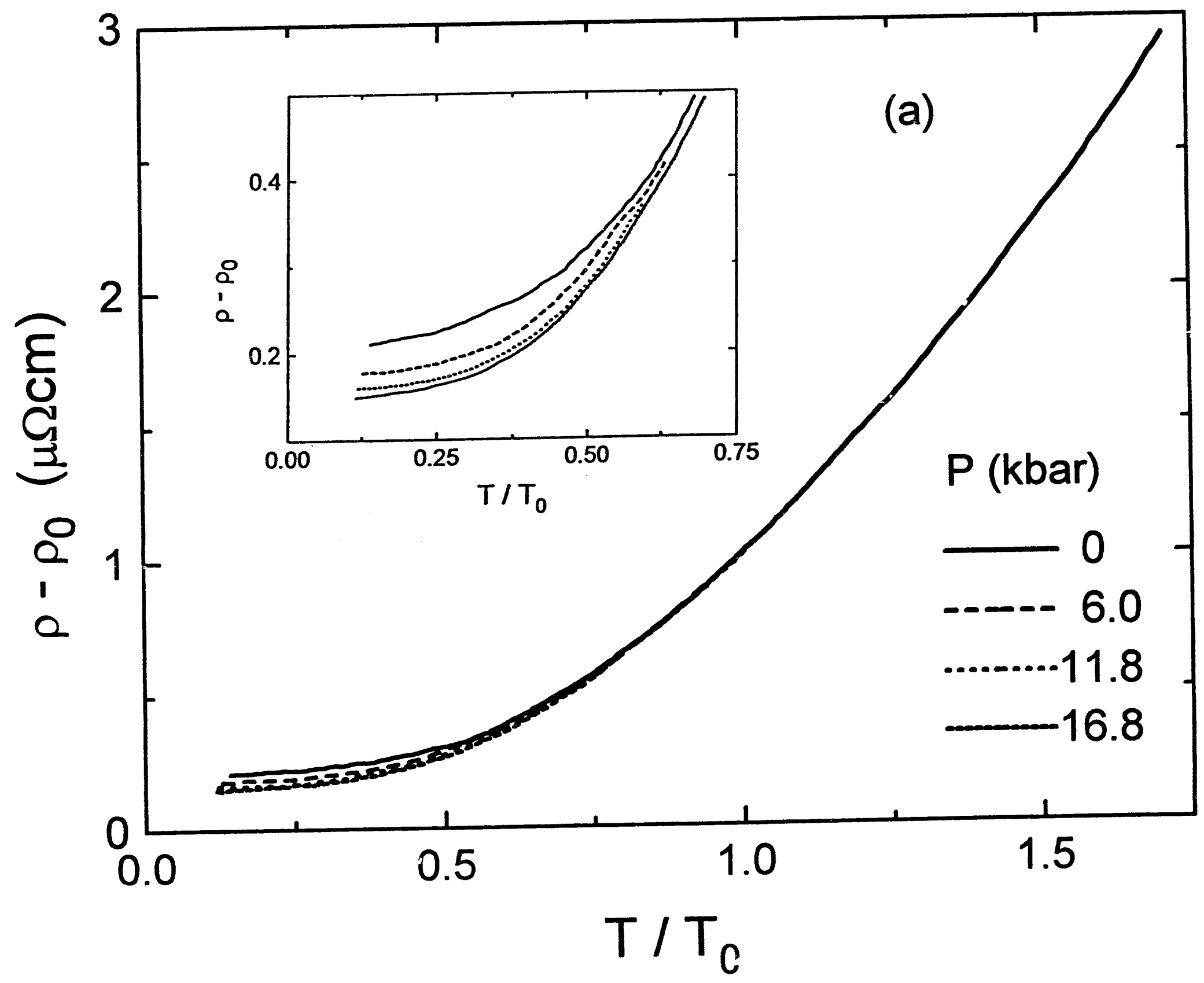




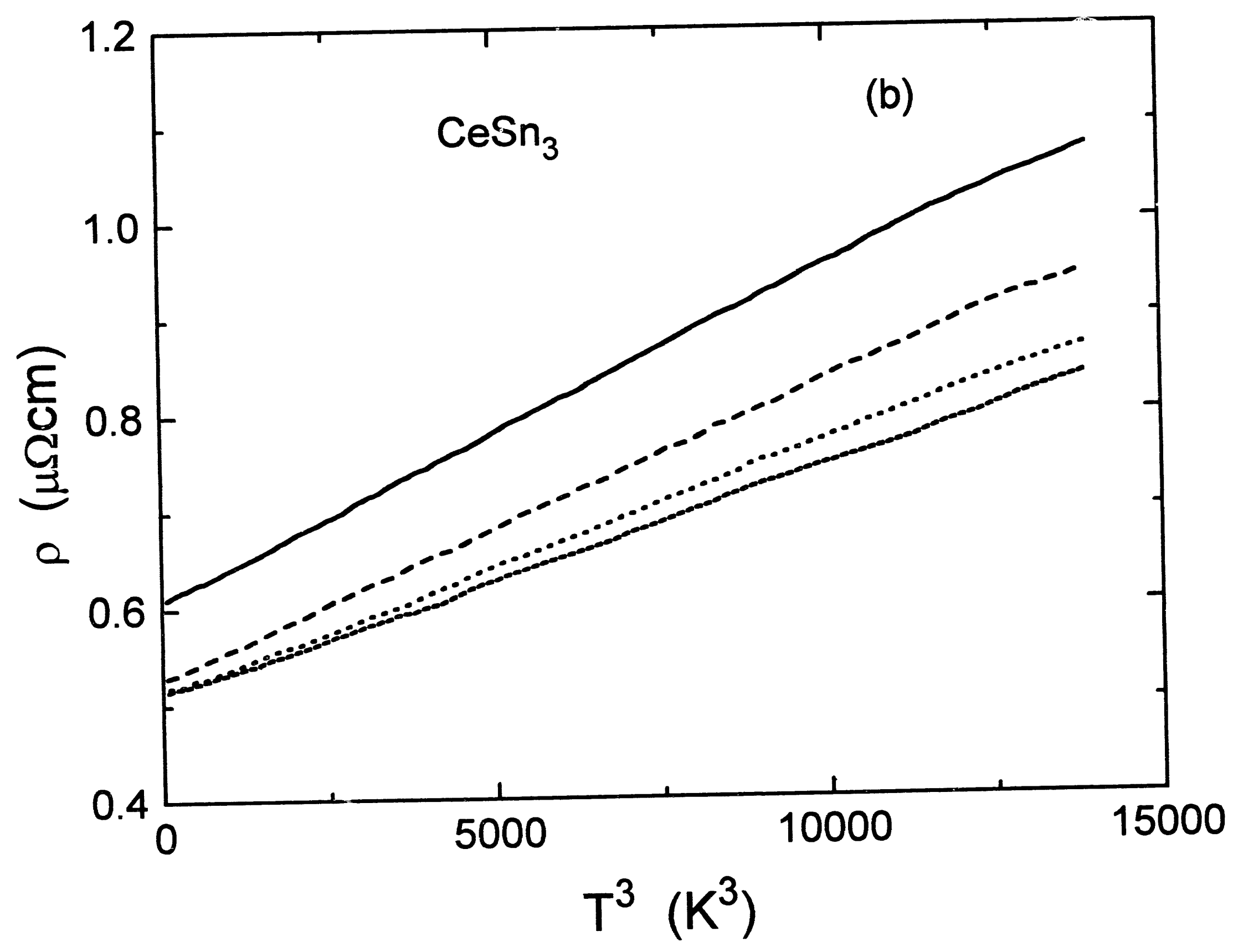




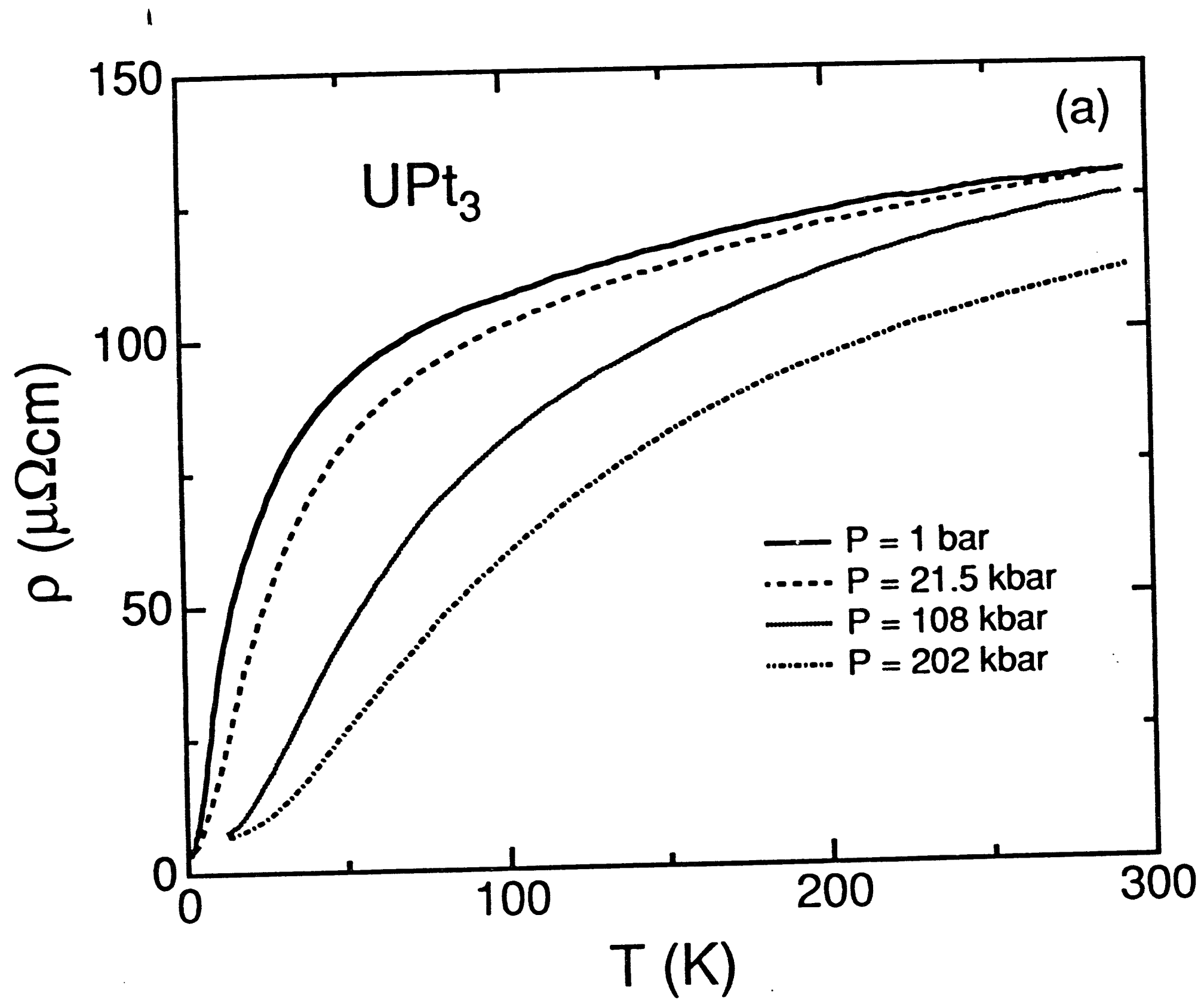




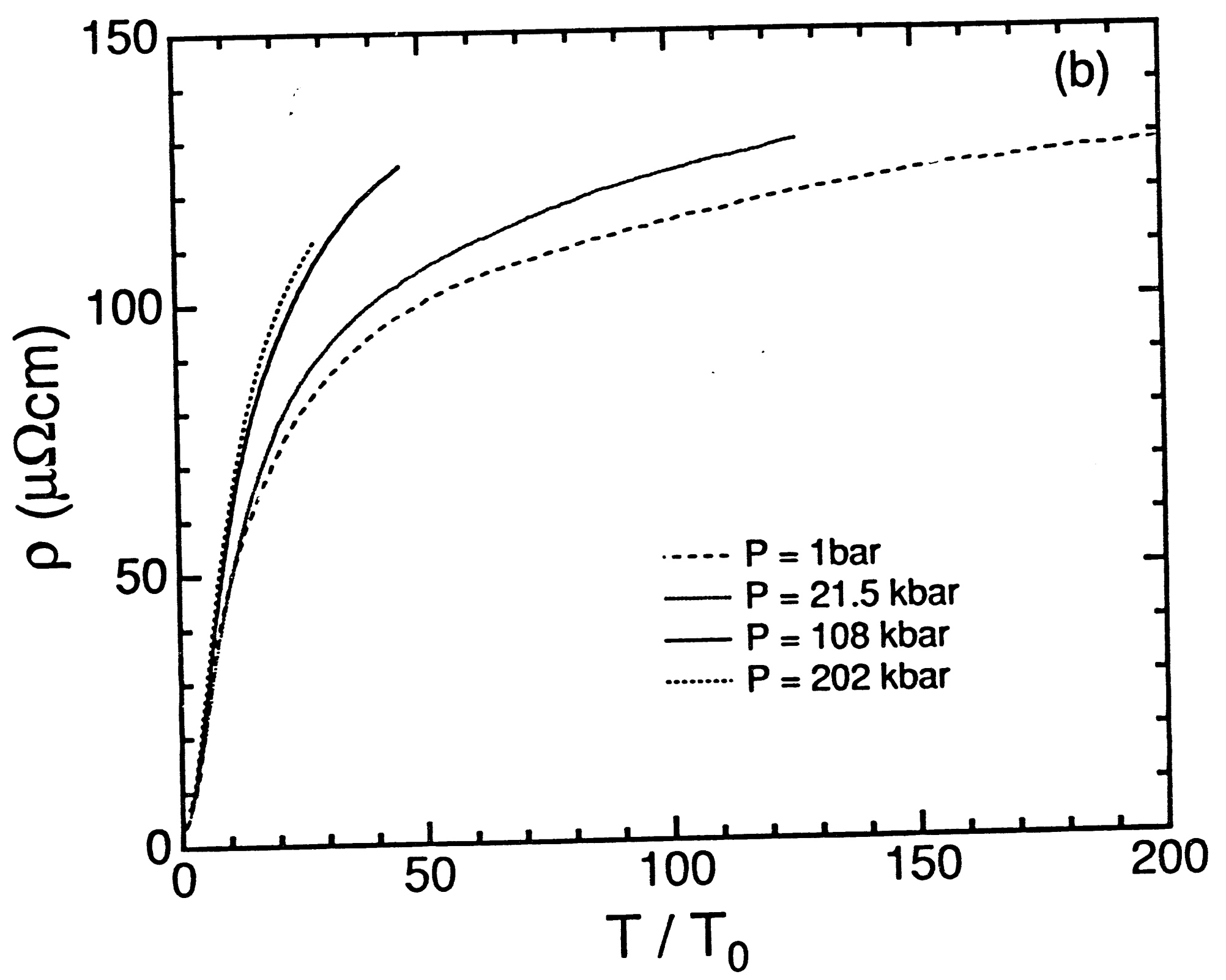




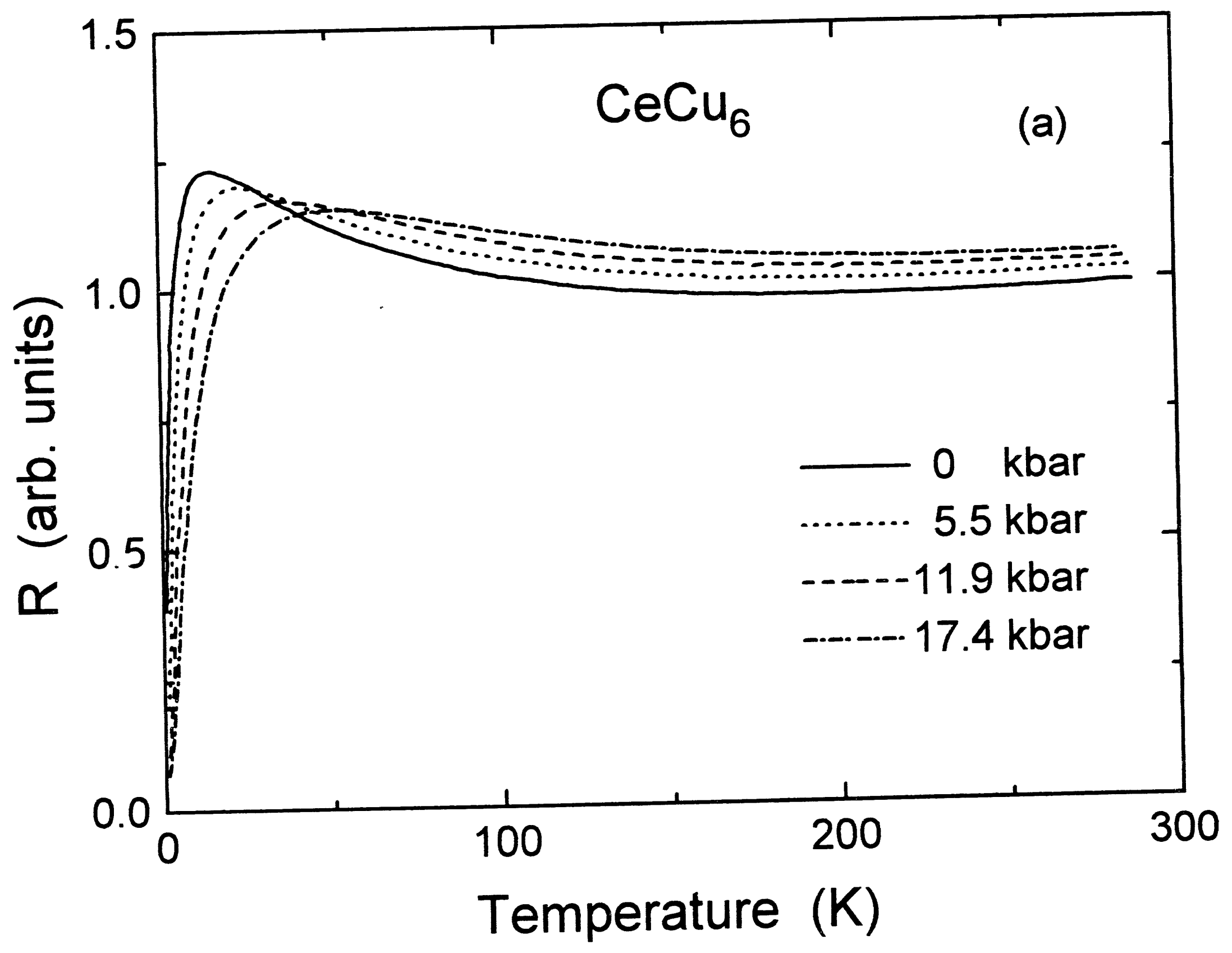




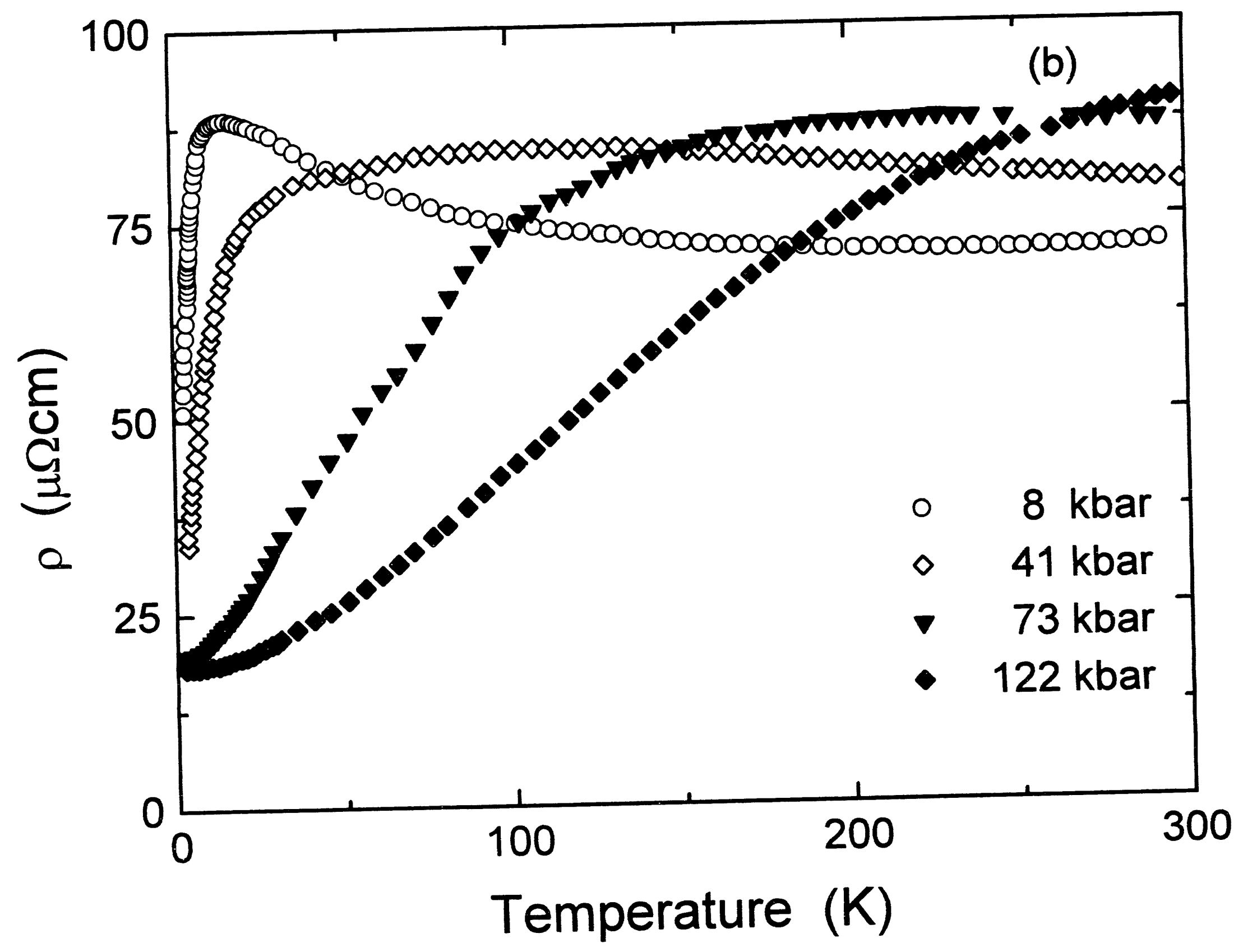




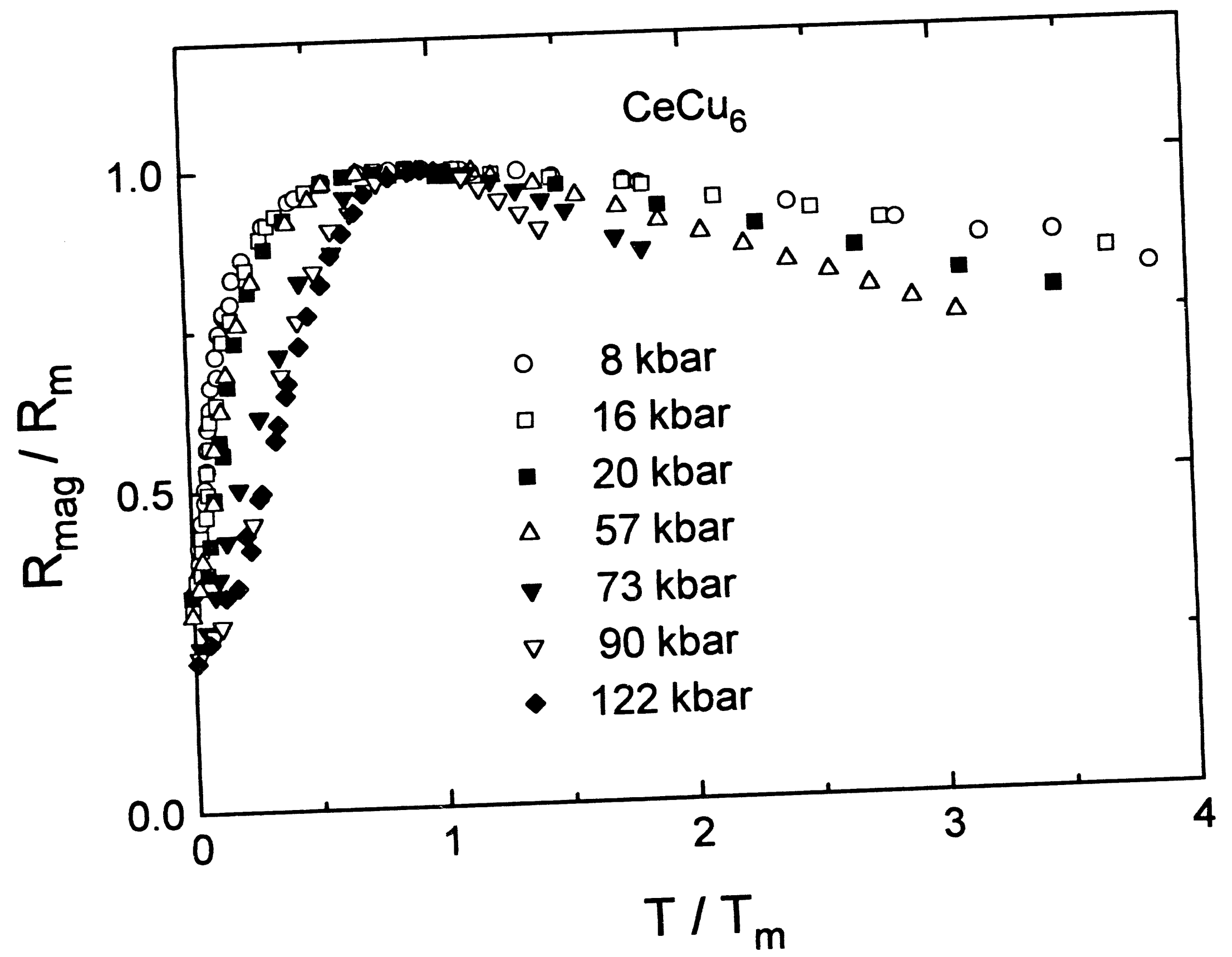




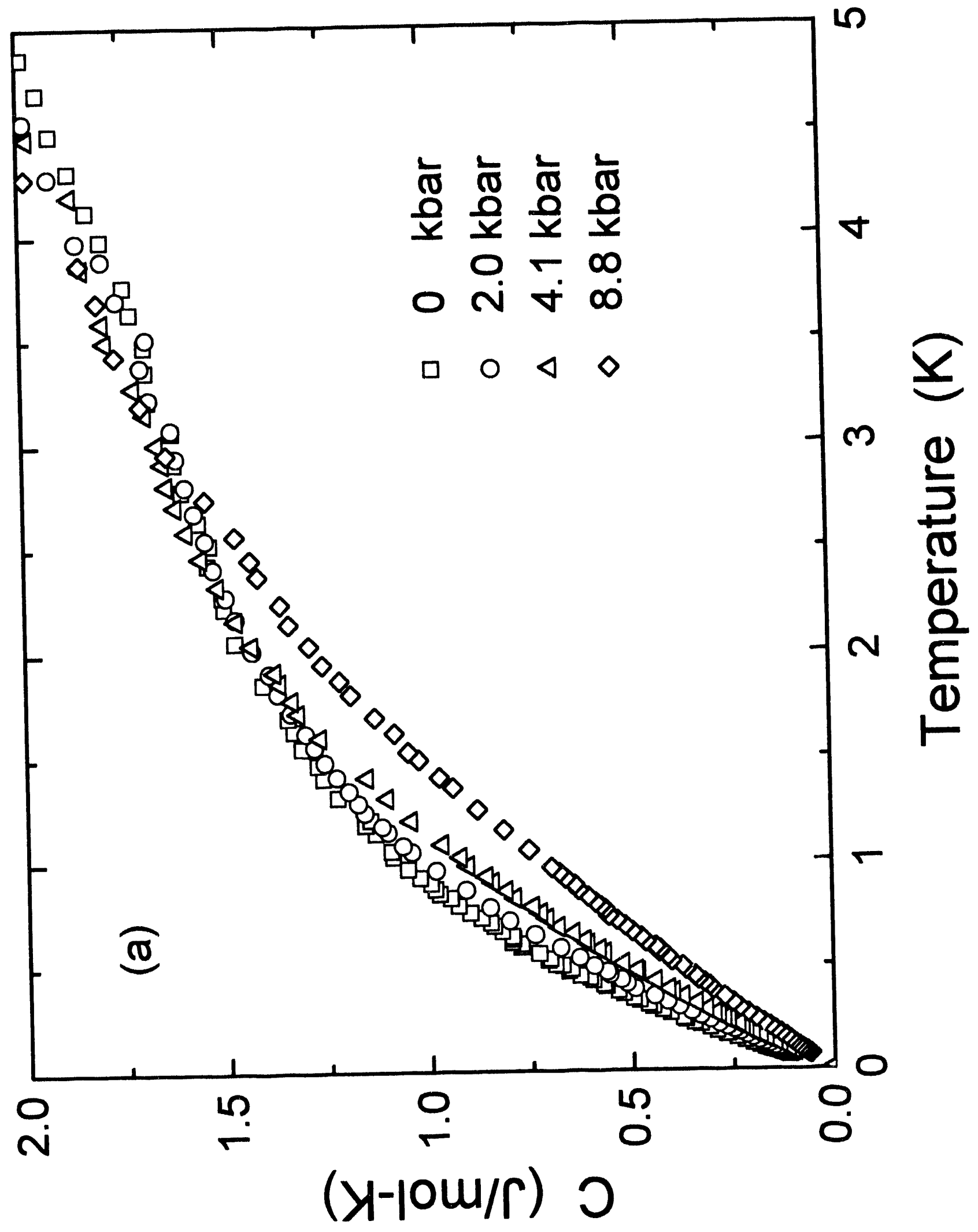




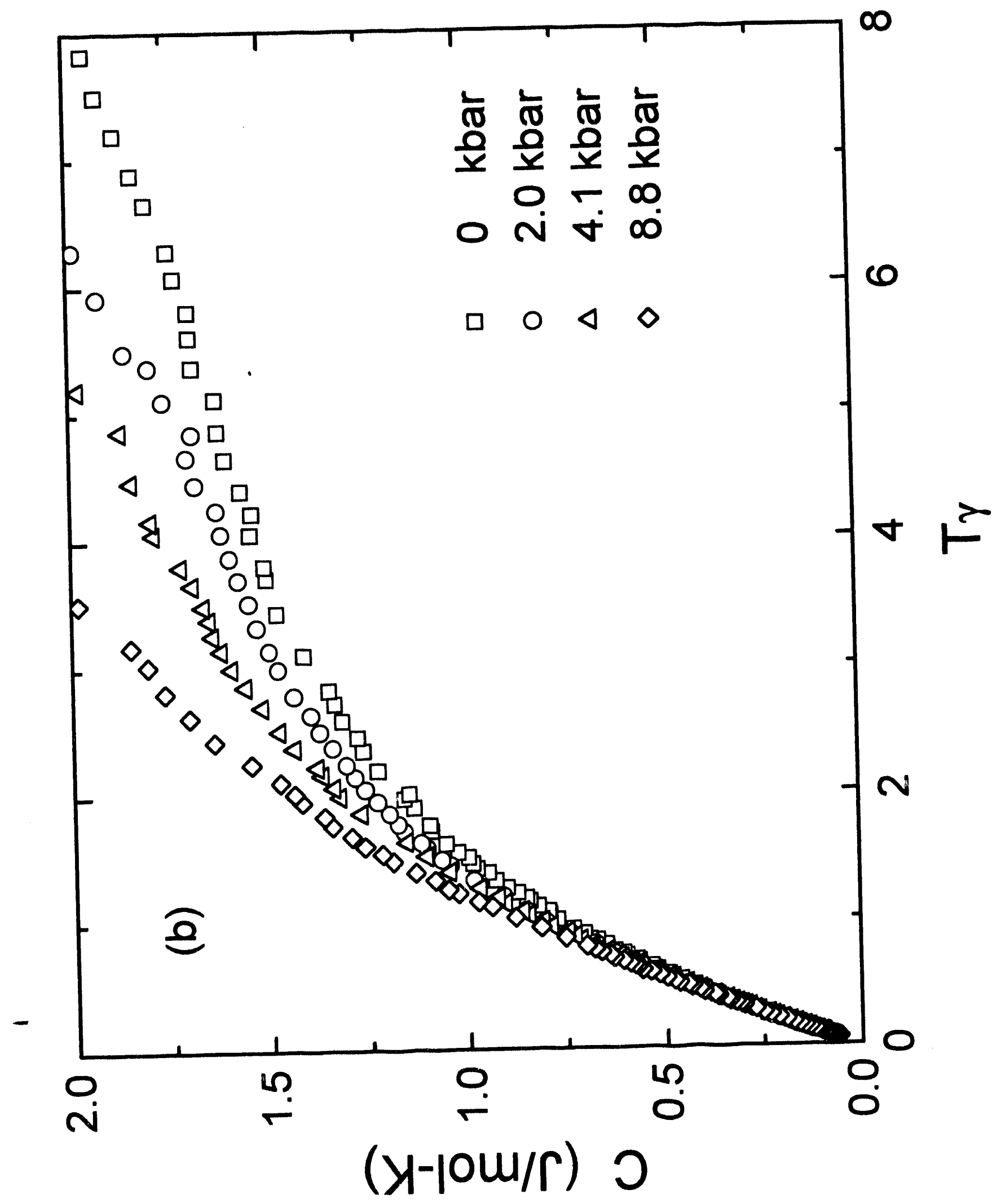




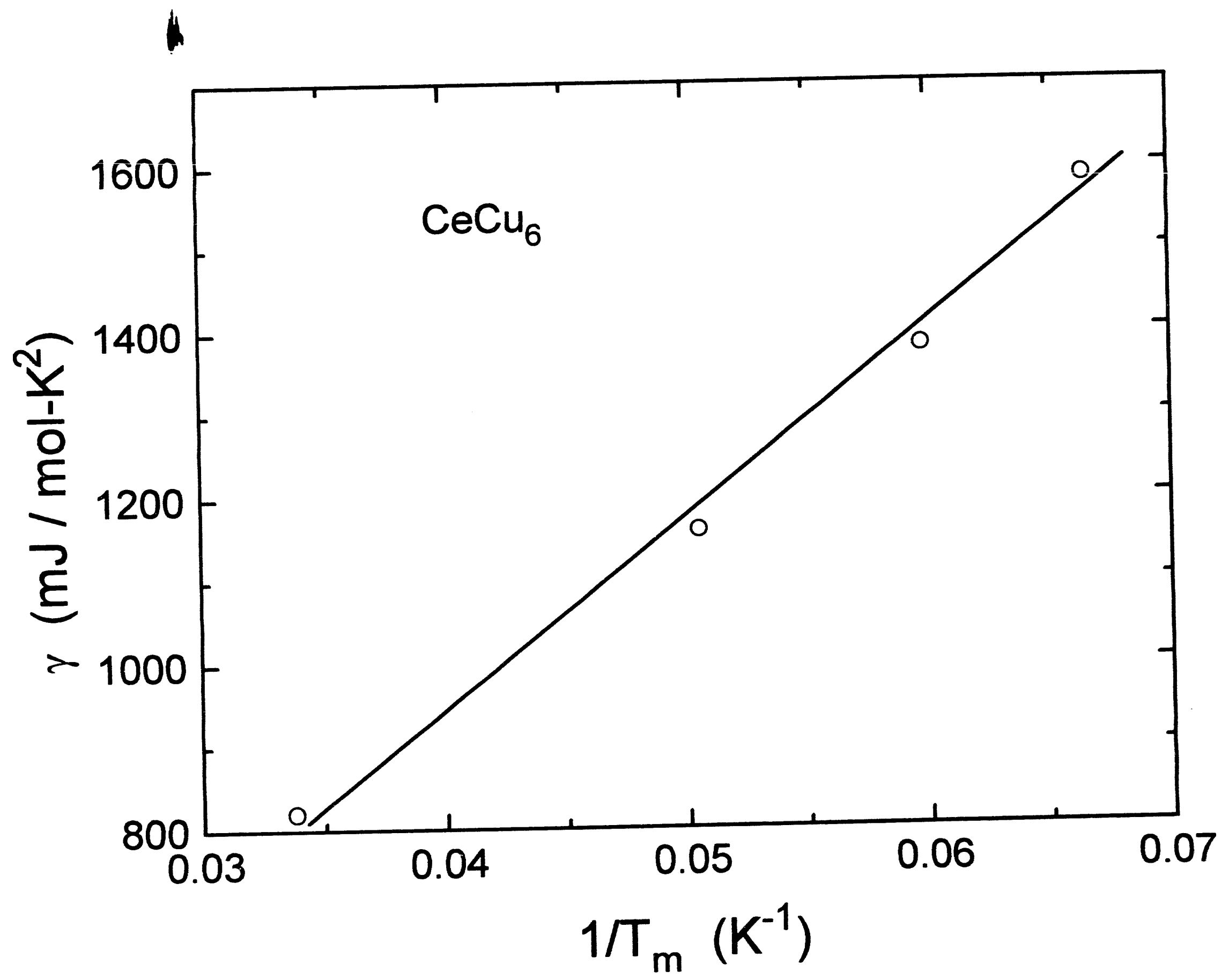




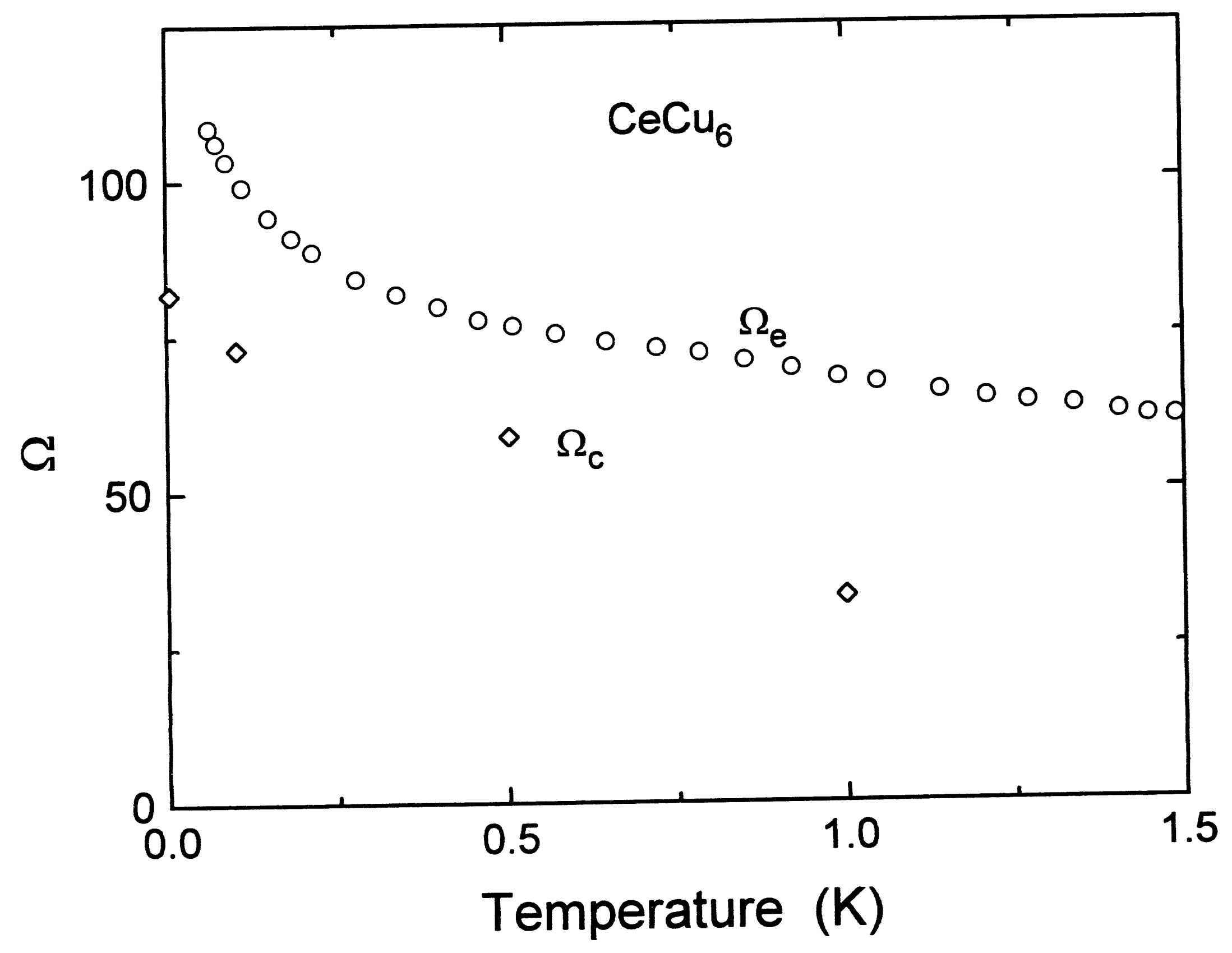





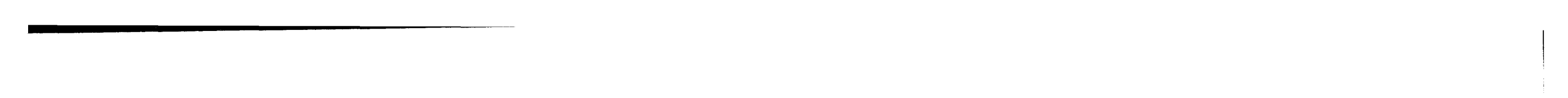

1

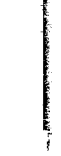

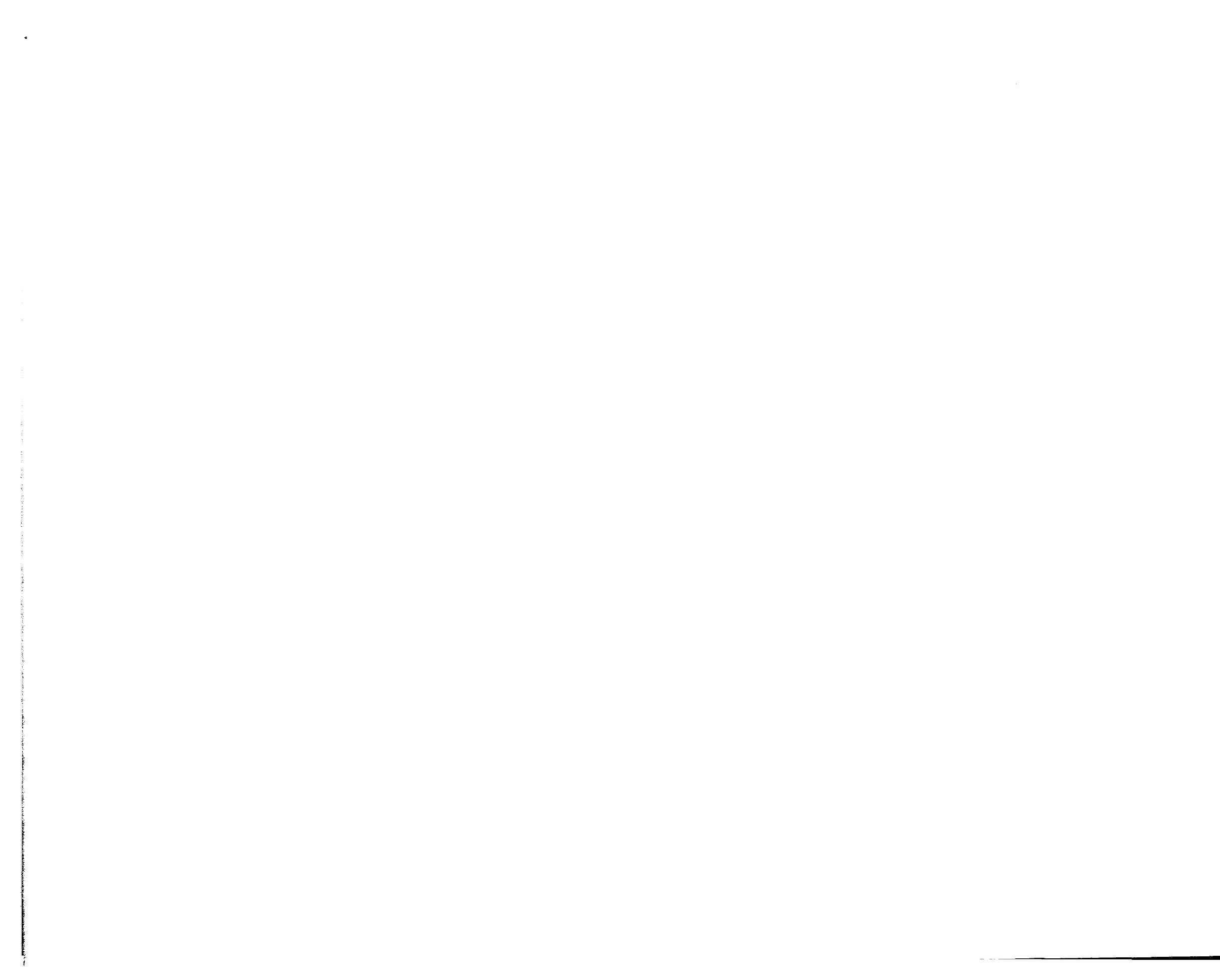

.
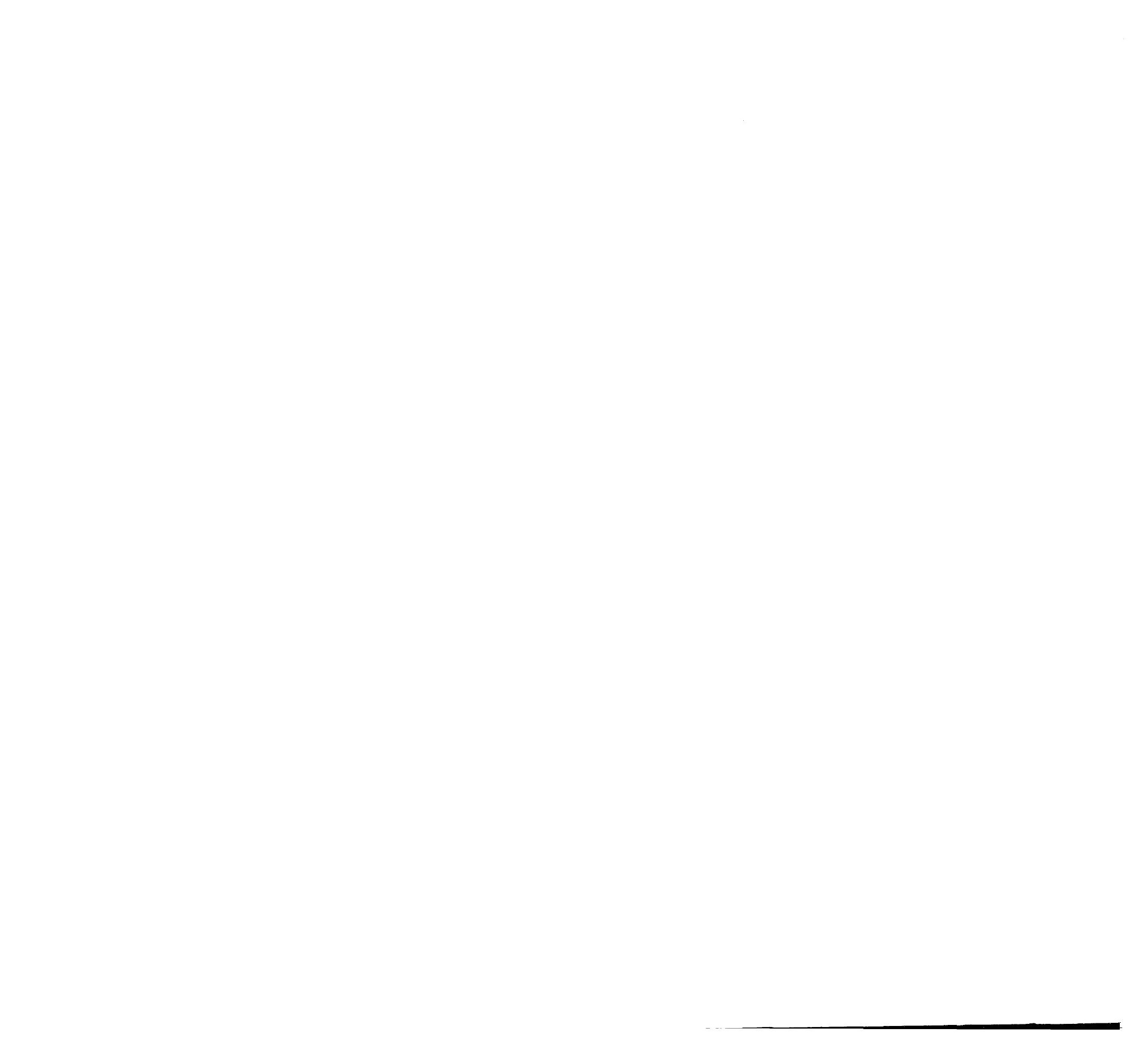